\title{
Cacti and filtered distributive laws
}

\author{
VLADIMIR DOTSENKO \\ JAMES GRIFFIN
}

\begin{abstract}
Motivated by the second author's construction of a classifying space for the group of pure symmetric automorphisms of a free product, we introduce and study a family of topological operads, the operads of based cacti, defined for every pointed simplicial set $(Y, p)$. These operads also admit linear versions, which are defined for every augmented graded cocommutative coalgebra $C$. We show that the homology of the topological operad of based $Y$-cacti is the linear operad of based $H_{*}(Y)$-cacti. In addition, we show that for every coalgebra $C$ the operad of based $C$-cacti is Koszul. To prove the latter result, we use the criterion of Koszulness for operads due to the first author, utilising the notion of a filtered distributive law between two quadratic operads. We also present a new proof of that criterion, which works over a ground field of arbitrary characteristic.
\end{abstract}

18D50; 20L05, 16S15

\section{Introduction}

One of the most famous algebraic operads of topological origin is the operad of Gerstenhaber algebras, which is the homology operad of the topological operad of little 2-disks (Cohen, Lada and May [9], and Getzler and Jones [16]). The $k^{\text {th }}$ component of the operad of little 2-discs is homotopy equivalent to the configuration space of $k$ ordered points in $\mathbb{R}^{2}$ whose fundamental group is the pure braid group on $k$ strands. One natural way to generalise braid groups is to consider configurations of subsets that have more interesting topology than points. The simplest example of these "higher-dimensional" versions of braid groups is given by "groups of loops", the $n^{\text {th }}$ one being the group of motions of $n$ unknotted unlinked circles in $\mathbb{R}^{3}$ bringing each circle to its original position. Alternatively, these groups can be viewed as groups of pure symmetric automorphisms of the free group with $n$ generators, that is, automorphisms sending each generator to an element of its conjugacy class. The integral cohomology of these groups was computed by Jensen, McCammond and Meier in [19]; that paper also contains references and historical information on this group. The description of the cohomology algebras in [19] looks very similar to that for pure braid 
groups (Arnol'd [2]). Moreover, as a symmetric collection, the collection of cohomology algebras is isomorphic to Com $\circ$ PreLie $_{1}$, which bears striking resemblance with the isomorphism $e_{2} \simeq$ Com $\circ \mathrm{Lie}_{1}$ for the operad of Gerstenhaber algebras. However, there is no natural operad structure on the collection of homology groups of the groups of loops. One way to repair that is to generalise the operad of little disks more directly, and consider configurations of unknotted and unlinked filled in little tori $S^{1} \times D^{2}$ inside a torus. Some of our results may be viewed as a first approximation towards understanding the topology of that operad.

In [18], the second author computed the cohomology of the groups of pure symmetric automorphisms in a different way, as a particular case of a much more general result: for an arbitrary $n$-tuple of groups $\left(G_{1}, \ldots, G_{n}\right)$, he computed the cohomology of the Fouxe-Rabinovitch group $\operatorname{FR}(G)$ of partial conjugation automorphisms of the free product $G=G_{1} * \cdots * G_{n}$. For that, he used a construction of a classifying space of that group via a moduli space of "cactus products" of the classifying spaces $Y_{i}=B G_{i}$. In the case when $G_{1}=G_{2}=\cdots=G_{n}$, these spaces form a symmetric collection, but alas do not form a topological operad either. However, it turns out that they admit a slight modification that carries a structure of a topological operad; the required change is that one of the spaces $Y_{i}$ is chosen as the base and is required to sit at the root of each cactus. We call the modified space the space of based $Y$-cacti. The goal of this paper is to understand the algebra and topology of this operad.

For homology with coefficients in a field, we show that the homology operad of the operad of based $Y$-cacti is obtained from the homology coalgebra of $Y$ by a formal algebraic procedure that only uses the augmentation and the coproduct; thus, it is defined for every graded cocommutative coalgebra $C$, not necessarily the homology coalgebra of a topological space. Remarkably, for every coalgebra $C$ this defined operad is Koszul. To prove that, we use filtered distributive laws between operads, as defined by the second author in [10]. One immediate consequence of our results is that, for $Y=S^{1}$, the homology operad of based $Y$-cacti is isomorphic, as an $\mathbb{S}$-module, to Perm $\circ$ PreLie $_{1}$, which, given that the operad of associative permutative algebras Perm encodes commutative algebras with additional structure, may be naturally thought of as an "operad-compatible improvement" of the result of [19] mentioned above.

Our constructions are defined over a field of arbitrary characteristic, and our results on operads of based cacti hold in that generality. However, even the distributive law criterion for Koszulness, let alone its filtered generalisation, has only been available in zero characteristic, since the known proofs (Dotsenko [10] and Vallette [35]) rely on the Künneth formula for symmetric collections. Using the shuffle operads technique (Dotsenko and Khoroshkin [12; 13]), we were able to obtain a characteristic-independent proof of this criterion. 
The paper is organised as follows. In Section 2, we recall necessary background information that we use throughout the paper. In Section 3, we define the topological operads of based cacti and discuss its connections both with automorphism groups of free products and with other known topological operads. The homology operad for the operad of based cacti is computed in Section 4. In Section 5, we discuss filtered distributive laws between quadratic operads. Section 6 shows how to use filtered distributive laws to prove the Koszul property for the linear operads of based cacti, and also discusses its applications to the operad of post-Lie algebras and the operad of commutative tridendriform algebras.

\section{Acknowledgements}

This work originated from a discussion of results of [19] the authors had when they first met at the workshop "Algebra, Combinatorics and Dynamics" in Belfast in 2009; we want to thank Natalia Iyudu and Stanislav Shkarin for an invitation to attend that workshop. We also wish to thank Muriel Livernet for pointing out the reference Méndez [31].

\section{Trees, coalgebras, operads}

All "linear" objects in this paper (algebras, coalgebras, operads) are objects of a certain concrete (equipped with a faithful functor to sets) closed symmetric monoidal category $(\mathcal{C}, \otimes, \sigma, \mathbb{I})$, usually the category Vect of vector spaces or the category gVect of graded vector spaces (over some field $\mathbb{k}$; unless otherwise specified, we do not make any assumptions on its characteristic). Whenever appropriate, we assume vector spaces to be finite-dimensional, or possessing an additional $\mathbb{N}$-grading with finite-dimensional homogeneous components; this allows to approach tensor constructions and duals with ease, freely passing between an algebra and its dual coalgebra, etc.

\subsection{Y-labelled trees}

A tree is an acyclic connected graph and a rooted tree is a tree with a chosen vertex, the root. A rooted tree may be directed: every edge $\{v, w\}$ may be oriented to $\overrightarrow{v w}$ in such a way that the minimal path from $w$ to the chosen vertex contains $\{v, w\}$. By the acyclicity of the tree this must hold for exactly one of the choices $\overrightarrow{v w}$ and $\overrightarrow{w v}$. The edges may be seen to be directed "away from the roots". We denote by $E(T)$ the set of edges of a tree $T$.

Suppose that $T$ is a tree with vertex set $V$. Let $\mathbf{Y}=\left(Y_{i}\right)_{i \in V}$ be a $V$-tuple of sets. Then a $\mathbf{Y}$-tree is a rooted tree with an edge labelling where the edge $\overrightarrow{i j}$ is labelled 
by an element of $Y_{i}$. As shorthand we define a $Y$-tree for a set $Y$ to be a $\mathbf{Y}$-tree where the $V$-tuple $\mathbf{Y}$ is constantly $Y$. Then the edge labelling is a map from the edge set $E$ to the set $Y$. Meanwhile a $Y$-forest is a $\mathbf{Y}$-tree where $\mathbf{Y}$ is the $V$-tuple with $Y_{0} \cong\{p\}$, where 0 is the root vertex and $Y_{v} \cong Y$ for any other vertex. The naming makes sense because by removing the root 0 and all adjacent edges we are left with a disjoint union of $Y$-trees; the root of each tree is the unique vertex adjacent to 0 and the edge labelling is inherited.

To a rooted tree $T$ we define the level $l(T)$ to be the number of non-trivial directed paths in $T$. So for a corolla with root 1 and $k-1$ other vertices the level is $k-1$, and for a tree with root 1 and edges $\overrightarrow{i(i+1)}$ for $i=1, \ldots, k-1$ the level is $k(k-1) / 2$. The level allows one to filter the set of $\mathbf{Y}$-trees.

\subsection{Coalgebras}

A coalgebra is an object $C$ of $\mathcal{C}$ equipped with a comultiplication $\Delta: C \rightarrow C \otimes C$ and a counit $\epsilon: C \rightarrow \mathbb{I}$ satisfying the conventional coassociativity and counit axioms. For the comultiplication, we often use Sweedler's notation $\Delta(c)=\sum c_{(1)} \otimes c_{(2)}$. An augmented coalgebra is a coalgebra $C$ equipped with a coalgebra homomorphism $\gamma: \mathbb{I} \rightarrow C$ such that $\epsilon \gamma=1$. A cocommutative coalgebra is a coalgebra satisfying $\sigma \Delta=\Delta$. Our main focus will be on graded augmented cocommutative coalgebras, that is, augmented cocommutative coalgebras in gVect. The main source of such coalgebras relevant for our purposes is topology: the homology coalgebra of a pointed simplicial set $(Y, p)$ is a graded augmented cocommutative coalgebra. An augmented coalgebra in Vect or gVect naturally splits into a direct sum of vector spaces $C=\mathbb{k} \mathbb{1} \oplus \bar{C}$, where $\mathbb{1}=\gamma(1), \bar{C}=\operatorname{ker}(\epsilon)$.

\subsection{Operads}

We refer the reader to Loday and Vallette [28] for further information on operads, and to [12] for further details on Gröbner bases. In this section we only recall the key notions used throughout the paper. By an operad in a symmetric monoidal category $(\mathcal{C}, \otimes, \sigma, \mathbb{I})$ we mean a monoid in one of the two monoidal categories: the category of symmetric $\mathcal{C}$-collections equipped with the composition product or the category of nonsymmetric $\mathcal{C}$-collections equipped with the shuffle composition product. The former kind of monoids is referred to as symmetric operads; the latter, as shuffle operads. In particular, we assume that our collections are reduced, that is, are functors from the category of nonempty finite sets (respectively, nonempty finite ordered sets) to $\mathcal{C}$ (note that the shuffle composition product is only defined for reduced collections). A good rule of thumb is that all operads defined in this paper are symmetric operads, but 
for computational purposes it is useful to treat them as shuffle operads, applying the forgetful functor from the category of symmetric collections to the category of nonsymmetric collections. (We denote the result of applying the forgetful functor to a collection $\mathscr{O}$ by $\mathscr{O}^{f}$ ). This does not lose any information except for the symmetric group actions, since the forgetful functor is monoidal and one-to-one on isomorphism classes of objects, and therefore for tasks that can be formulated without the symmetric group actions, eg computing bases and dimensions of components, proving the Koszul property etc, we can choose arbitrarily whether to work with a symmetric operad or with its shuffle version. In the "geometric" setting, $\mathcal{C}$ will usually be the category of sets, or simplicial sets or pointed simplicial sets, and in the "linear" setting it will usually be the category of vector spaces (in which case symmetric collections are usually called $\mathbb{S}$-modules), or the category of graded vector spaces or chain complexes (in which case symmetric collections are called differential graded $\mathbb{S}$-modules). A linear symmetric operad can also be thought as of collection of spaces of operations of some type, and therefore can be defined via its category of algebras, ie vector spaces where these operations act, via identities between operations acting on a vector space.

In the linear setting, a very useful technical tool for dealing with (shuffle) operads is given by Gröbner bases. More precisely, similarly to associative algebras, operads can be presented via generators and relations, that is, as quotients of free operads $\mathscr{F}(\mathscr{V})$, where $\mathscr{V}$ is the space of generators. The free shuffle operad generated by a given nonsymmetric collection admits a basis of "tree monomials", which can be defined combinatorially; a shuffle composition of tree monomials is again a tree monomial. In addition to the "arity" of elements of a free operad, there is the notion of weight, a generalisation of the word-length grading in free associative algebras: we define the weight of a tree monomial as the number of generators used in this tree monomial. Weight is well behaved under composition: when composing several tree monomials, the weight of the result is equal to the sum of their weights. For an arbitrary operad $\mathscr{O}=\mathscr{F}(\mathscr{V}) /(\mathscr{R})$ whose relations $\mathscr{R}$ are weight-homogeneous, the weight descends from the free operad $\mathscr{F}(\mathscr{V})$ on $\mathscr{O}$; the subcollection of $\mathscr{O}$ consisting of all elements of weight $k$ is denoted by $\mathscr{O}_{(k)}$.

There exist several ways to introduce a total ordering of tree monomials in such a way that the operadic compositions are compatible with that total ordering. There is also a combinatorial definition of divisibility of tree monomials that agrees with the naive operadic definition: one tree monomial occurs as a subtree in another one if and only if the latter can be obtained from the former by operadic compositions. A Gröbner basis of an ideal $I$ of the free operad is a system $S$ of generators of $I$ for which the leading monomial of every element of the ideal is divisible by one of the leading terms of elements of $S$. Such a system of generators allows one to perform "long 
division" modulo $I$, computing for every element its canonical representative. There exists an algorithmic way to compute a Gröbner basis starting from any given system of generators ("Buchberger's algorithm for shuffle operads").

A part of operad theory, which provides one of the most useful known tools to study homological and homotopical algebra for algebras over a given operad, is the Koszul duality for operads (Ginzburg and Kapranov [17]). Proving that a given operad is Koszul instantly provides a minimal resolution for this operad and gives a description of the homology theory and, in particular, the deformation theory for algebras over that operad, etc. There are a few general methods to prove that an operad is Koszul; one of the simplest and widely applicable methods $[12 ; 13]$ is to show that a given operad has a quadratic Gröbner basis (as a shuffle operad); this provides a sufficient (but not necessary) condition for Koszulness of an operad. If an operad is Koszul, it necessarily is quadratic, that is, can be presented using only weight-homogeneous relations of weight 2 .

The operads that serve as "building blocks" for operads considered throughout this paper are mostly well known: Com (commutative associative algebras), Lie (Lie algebras), As (associative algebras), Leib (Leibniz algebras; Loday [25]), Zinb (Zinbiel algebras; Loday [26]), Perm ((associative) permutative algebras; Chapoton [7]), NAP (nonassociative permutative algebras (Livernet [24]), closely related to "right-commutative magma" (Dzhumadil'daev and Löfwall [15])). All these operads are Koszul and have a quadratic Gröbner basis.

\subsection{Polynomial functors}

As we said before, some of our constructions exist both in a "geometric" and a "linear" setting, and are related to each other via the homology functor (which assigns to a topological space $Y$ the graded cocommutative coalgebra $H_{*}(Y)$ ). To make additional structures transfer easily, we use basic concepts from the theory of polynomial functors. A polynomial functor is a notion that categorifies the notion of a polynomial, and more generally of a formal power series. Polynomial functors provide a useful uniform language to deal with categorical constructions that have "a polynomial flavour", eg when computing sums and products in appropriate categories over specified sets indexing summands/factors in a way that keeps track of the intrinsic structure of the indexing sets.

In precise words, a map of sets

$$
p: E \rightarrow B
$$

gives rise to a functor $F_{p}$ : Set $\rightarrow$ Set defined by the formula

$$
F_{p}(X)=\sum_{b \in B} X^{f^{-1}(b)} .
$$


Here one can replace Set by another category where all the appropriate notions make sense. These functors were referred to as polynomial functors in Moerdijk and Palmgren [33], and are called polynomial functors in one variable in more recent literature. For a systematic introduction to polynomial functors, we refer the reader to the paper [23] and the notes [22] by Kock, Joyal, Batanin and Mascari that reflect the state of the art.

\section{The operad of cacti}

\subsection{The operad NAP $\mathbf{P}_{Y}$}

Let $Y$ be a set and let $\operatorname{NAP}_{Y}(n)$ be the set of $Y$-trees with vertex set $\{1, \ldots, n\}$. When $Y$ is a singleton set this is just the set of rooted trees, which we denote $\mathrm{RT}(n)$. The symmetric group $\mathbb{S}_{n}$ acts on $\operatorname{NAP}_{Y}(n)$ via its action on the vertex set. For a given rooted tree the set of $Y$-labellings is equal to $\operatorname{Hom}(E, Y)=Y^{E}$. Since the number of edges of a tree on $\{n\}$ is always $n-1$, the set of $Y$-labellings is in turn isomorphic to $Y^{n-1}$. Hence

$$
\operatorname{NAP}_{Y}(n) \cong \coprod_{T \in \operatorname{RT}(n)} Y^{n-1}
$$

Now let $T_{1} \in \operatorname{NAP}_{Y}(n)$ and $T_{2} \in \operatorname{NAP}_{Y}(m)$ and $i \in[n]$. We may define a composition $T_{1} \circ_{i} T_{2} \in \operatorname{NAP}_{Y}(n+m-1)$ by first identifying the root of $T_{2}$ with the vertex $i$ in $T_{1}$. This is a tree and may be rooted by taking the root of $T_{1}$. The edge set is equal to the union $E\left(T_{1}\right) \amalg E\left(T_{2}\right)$ of the edge sets of $T_{1}$ and $T_{2}$ and so one inherits an edge labelling by elements of $Y$. It has the vertex set

$$
\{1, \ldots, i-1\} \amalg\{1, \ldots, m\} \amalg\{i+1, \ldots, n\} .
$$

We then relabel the vertices by elements of $[n+m-1]$ using the isomorphism that fixes $\{1, \ldots, i-1\}$, shifts the set $\{1, \ldots, m\}$ to $\{i, \ldots, m+i-1\}$ and shifts $\{i+1, \ldots, n\}$ to $\{m+i, \ldots, m+n-1\}$. This gives a rooted $Y$-tree on the vertex set $\{1, \ldots, n+m-1\}$, and so an element $T_{1} \circ_{i} T_{2} \in \operatorname{NAP}_{Y}(n+m-1)$.

Proposition 3.1 Let $Y$ be a set. Then, the maps

$$
\circ_{i}: \operatorname{NAP}_{Y}(n) \times \operatorname{NAP}_{Y}(m) \rightarrow \operatorname{NAP}_{Y}(n+m-1)
$$

for $i=1, \ldots, n$ give the collection $\operatorname{NAP}_{Y}$ an operad structure. The operad is generated by its binary operations

2
$\uparrow$
$1 y$$\quad$ and $\begin{array}{r}1 \\ \uparrow \\ 2 z\end{array} \quad$ for $y, z \in Y$,


and these satisfy the quadratic relation

$$
\begin{array}{ccc}
2 & & 2 \\
\uparrow & \circ_{1} & \uparrow \\
1_{y} & & 1_{z}
\end{array}=\left(\begin{array}{ccc}
2 & & 2 \\
\uparrow & \circ_{1} & \uparrow \\
1_{z} & & 1_{y}
\end{array}\right) \cdot(23),
$$

where (23) is the transposition swapping the labels 2 and 3.

Proof Let $T_{1}, T_{2}$ and $T_{3}$ be $Y$-trees in $\operatorname{NAP}_{Y}\left(n_{1}\right), \operatorname{NAP}_{Y}\left(n_{2}\right)$ and $\operatorname{NAP}_{Y}\left(n_{3}\right)$, respectively. Let $i<j \in\left[n_{1}\right]$ and $k \in\left[n_{2}\right]$; we must show that the two associativity relations hold:

$$
\begin{aligned}
& \left(T_{1} \circ_{j} T_{2}\right) \circ_{i} T_{3}=\left(T_{1} \circ_{i} T_{3}\right) \circ_{j+n_{3}-1} T_{2}, \\
& T_{1} \circ_{i}\left(T_{2} \circ_{k} T_{3}\right)=\left(T_{1} \circ_{i} T_{2}\right) \circ_{k+i-1} T_{3} .
\end{aligned}
$$

In both cases we are gluing together trees by identifying vertices - in the first we identify the roots of $T_{2}$ and $T_{3}$ with the vertices $j$ and $i$ of $T_{1}$, respectively - whilst in the second the root of $T_{2}$ is joined to vertex $i$ of $T_{1}$ and the root of $T_{3}$ is identified with vertex $k$ of $T_{2}$. The only complication is that when two trees are composed their vertices are renumbered: this change is taken into account in the right-hand side of each equation. In both cases the edge set of the resulting tree is the union of the edge sets of the three component trees, hence the $Y$-labellings on both sides of each equation are equal. It remains to make the routine check that the vertex labels in each side of each equation agree; this is no more complicated than the analogous check in the associative operad.

Now we show that the operad is generated by operations of arity 2 . Let $T \in \operatorname{NAP}_{Y}(n)$ be a $Y$-tree and let $\overrightarrow{i j}$ be a leaf of $T$; let $y$ be the label of $\overrightarrow{i j}$. By applying a permutation if necessary we may assume that $i=n-1$ and $j=n$. Let $T^{\prime}$ be the $Y$-tree in $\operatorname{NAP}_{Y}(n-1)$ given by removing the edge $\overrightarrow{(n-1) n}$ and the vertex $n$. Then we have that:

$$
T=T^{\prime} \circ_{n-1} \begin{gathered}
2 \\
\uparrow \\
1_{y}
\end{gathered}
$$

Therefore any $Y$-tree may be written as compositions of trees with two vertices and a permutation and so $\mathrm{NAP}_{Y}$ is generated in arity 2 .

The relation (3.1.5) is to seen to hold by evaluating each side of the equation to find the same $Y$-tree:

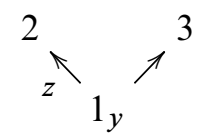


The above proposition gives quadratic relations in the binary generators; the Corollary 6.7 will show that these suffice to present the operad.

Remark 3.2 The operads $\operatorname{NAP}_{Y}$ are functorial in sets $Y$; in fact $\operatorname{NAP}_{(-)}(n)$ is a polynomial functor given by the map

$$
\coprod_{T \in \mathrm{RT}(n)} E(T) \rightarrow \mathrm{RT}(n) .
$$

Both the operad maps and the proof above work on the level of the polynomial itself, hence for any appropriate category one may use the polynomial to give a family of operads $\mathrm{NAP}_{(-)}$. For instance this means that if $Y$ is also equipped with a topology then $\mathrm{NAP}_{Y}$ is a topological operad. In Section 4 we will consider the operads $\mathrm{NAP}_{D}$ where $D$ is a graded vector space.

Remark 3.3 When $Y$ is a single point $\{p\}$, the operad $\operatorname{NAP}_{Y}$ is the usual operad NAP.

Let us finish this section with a few words on $\mathrm{NAP}_{Y}$-algebras. One convenient way to think of such an algebra $A$ is via the "right regular module", since the defining relations say that all the operators

$$
a \mapsto \underset{1}{2} \underset{1}{2}\left(a, a^{\prime}\right)
$$

on $A$ given by multiplying by a particular element $a^{\prime}$ on the right (such a gadget is defined for each $y \in Y$ and each $a^{\prime} \in A$ ) commute with each other. Somewhat more precisely, let $A$ be an object in a closed symmetric monoidal category $\mathcal{C}$, and let

$$
f: Y \times_{\mathcal{C}} A \rightarrow \operatorname{Hom}_{\mathcal{C}}(A, A)
$$

be a morphism whose image is an abelian submonoid. Then $A$ is a $\mathrm{NAP}_{Y}$-algebra in $\mathcal{C}$ with the structure maps given by

$$
\underset{1}{y \uparrow}(a, b)=f(y, b)(a) .
$$

This way to approach $\mathrm{NAP}_{Y}$-algebras gives a source of examples based on Permalgebras with a family of maps as follows.

Example 3.4 Let $(A, \cdot)$ be a Perm-algebra in a closed symmetric monoidal category $\mathcal{C}$, and let $g: Y \rightarrow \operatorname{Hom}_{\mathcal{C}}(A, A)$ be a family of maps (note that these maps may be 
arbitrary, not necessarily algebra homomorphisms). Then $A$ is a $\mathrm{NAP}_{Y}$-algebra in $\mathcal{C}$ with the structure maps given by

$$
\underset{1}{2} \underset{1}{2}(a, b)=a \cdot g(y)(b) .
$$

One more observation we want to mention in this section is that the construction of the free NAP-algebra mentioned in [24] admits an immediate generalisation to the case of $\mathrm{NAP}_{Y}$-algebras: the free $\mathrm{NAP}_{Y}$-algebra in Set with the generating set $V$ admits a realisation as the set of $Y$-trees whose vertices carry labels from $V$, with the product defined in the same way as we defined the composition in the operad, that is,

$$
\underset{1}{2}(a, b)=\left(\begin{array}{cc}
2 \\
y \uparrow & \circ_{1} a \\
1 &
\end{array}\right) \circ_{2} b .
$$

In this composition the root of $b$ is joined to the root of $a$ by an edge labelled by $y$; the new root is taken to be the root of $a$.

\subsection{The operad of based cacti}

Let $V$ be a set and $\mathbf{Y}$ be a $V$-tuple of pointed sets. Let $T$ be a $\mathbf{Y}$-tree with root $r \in V$ and suppose that $\overrightarrow{i j}$ is an edge of $T$ where $i \neq r$. Suppose further that $\overrightarrow{i j}$ is labelled by the basepoint $p \in Y_{i}$. Then we say that $\overrightarrow{i j}$ is a reducible edge and that $T$ is reducible. Since $i$ is not the root there is a unique incoming edge $\overrightarrow{k i}$, which is labelled by some $y \in Y_{k}$. We define $T_{i j}$ to be the $\mathbf{Y}$-tree given by removing the edge $\overrightarrow{i j}$ and adding the edge $\overrightarrow{k j}$ with the label $y \in Y_{k}$. We say that $T_{i j}$ is a reduction of $T$.

Definition 3.5 Let $V$ be a finite set and $\mathbf{Y}$ be a $V$-tuple of pointed sets. Then the set of based $\mathbf{Y}$-cacti, BCACT $\mathbf{Y}$, is the set of equivalence classes of $\mathbf{Y}$-trees under the equivalence relation $\sim$, generated by $T \sim T_{i j}$ for any $T$ with a reducible edge $\overrightarrow{i j}$.

Now let $V_{0}$ be the set $V \cup\{0\}$ and let $\mathbf{Y}_{0}$ be the $V_{0}$-tuple given by adjoining $Y_{0}=\{p\}$ to the $V$-tuple $\mathbf{Y}$. Then we define the set of $\mathbf{Y}$-cacti, $\mathrm{CACT}_{\mathbf{Y}}$, to be the subset of $\operatorname{BCACT}_{\mathbf{Y}_{0}}$ consisting of the trees with root 0 .

Remark 3.6 For each $\mathbf{Y}$-cactus $T \in$ BCACT $_{\mathbf{Y}}$, one may define the set

$$
\mathbf{Y}(T)=\frac{\bigsqcup_{v \in V} Y_{v}}{y_{i j} \sim p_{j} \mid \overrightarrow{i j} \in E(T)},
$$


where $y_{i j} \in Y_{i}$ is the label of the edge $\overrightarrow{i j}$ and $p_{j}$ is the basepoint of $Y_{j}$. Note that this realisation is invariant across equivalences $T \sim T_{i j}$. If each set $Y_{i}$ is the realisation of a pointed, connected simplicial set then this space is homotopy equivalent to the wedge product of the spaces $Y_{i}$ for $i \in V$. These spaces are called cactus products and were studied by the second author in [18]. There it was shown that the set $\mathrm{CACT}_{\mathbf{Y}}$ of such products has interesting homotopical properties: in particular if the spaces $Y_{i}$ are classifying spaces for groups $G_{i}$ then $\mathrm{CACT}_{\mathbf{Y}}$ is a classifying space for the Fouxe-Rabinovitch group $\operatorname{FR}(G)$ of partial conjugation automorphisms of the free product $G=*_{i \in V} G_{i}$. Here is an example of a cactus product:

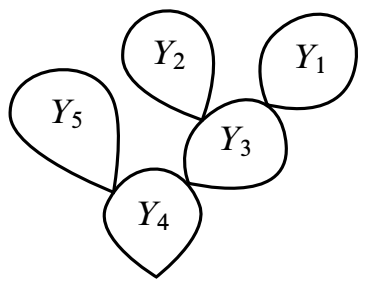

Note that if $v$ is the root of the tree $T$ then the space $Y_{v}$ must always be at the "base" of the diagram. The appearance of the diagram explains the term "based $\mathbf{Y}$-cactus". We also see the reason for adjoining a point space $Y_{0}$; this removes the base space; the space $Y_{0}$ acts as a basepoint.

Remark 3.7 Recall that the level of a rooted tree is the number of non-trivial directed paths. When $\overrightarrow{k i}$ and $\overrightarrow{i j}$ are edges of a rooted tree $T$, the rooted tree $T^{\prime}$ given by removing $\overrightarrow{i j}$ and then adding $\overrightarrow{k j}$ has strictly lesser level. Indeed if $P$ is the unique path joining vertices $v$ and $w$ in $T^{\prime}$, then there is a unique path joining $v$ and $w$ in $T$. But the number of paths in $T$ is strictly larger because there is a path joining $i$ and $j$ in $T$ but not in $T^{\prime}$. So for any $\mathbf{Y}$-tree $T$ one may use the reductions $T \sim T_{i j}$ repeatedly until there are no reducible edges remaining. Since the level reduces each time this process must terminate. It is easy to check that it does not matter what order in which the reductions $T \sim T_{i j}$ are applied because if $\overrightarrow{a b}$ and $\overrightarrow{c d}$ are two reducible edges then $\left(T_{a b}\right)_{c d}=\left(T_{c d}\right)_{a b}$. Hence for each $Y$-labelled tree there is a unique equivalent tree that cannot be reduced any further. Therefore $\mathrm{BCACT}_{\mathbf{Y}}$ is isomorphic to the set of irreducible $\mathbf{Y}$-trees.

Definition 3.8 Let $(Y, p)$ be a pointed set. For $n \geq 1$, we define the set $\operatorname{BCACT}_{Y}(n)$ to be the set of based cacti on the $n$-tuple $\mathbf{Y}=\left(Y_{i} \cong Y\right)_{i=1, \ldots, n}$. The action of $\mathbb{S}_{n}$ on $\{1, \ldots, n\}$ makes this into a symmetric collection. 
Theorem 3.9 Let $(Y, p)$ be a pointed set. The equivalence relation $\sim$ generated by reductions $T \sim T_{i j}$ is compatible with the operad maps of $\mathrm{NAP}_{Y}$. Hence the collection $\mathrm{BCACT}_{Y}$ has an operad structure inherited from $\mathrm{NAP}_{Y}$. Furthermore the equivalence relation $\sim$ is generated as an operad ideal by the single relation below.

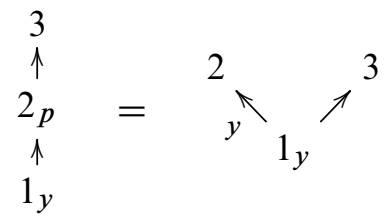

Proof Let $T \in \operatorname{NAP}_{Y}(n)$ be a $Y$-tree with reducible edge $\overrightarrow{i j}$; let $T^{\prime} \in \operatorname{NAP}_{Y}(m)$ be any other $Y$-tree. Then for any $k \in[n]$ and $l \in[m]$ the products

$$
T \circ_{k} T^{\prime} \text { and } T^{\prime} \circ_{l} T
$$

are both given by identifying vertices. The edge $\overrightarrow{i j}$ still exists in each product, although it may have been relabelled, to $\overrightarrow{i^{\prime} j^{\prime}}$, say. The label in $Y$ is still the point $p$. Furthermore $i^{\prime}$ is not the root in either product so $\overrightarrow{i^{\prime} j^{\prime}}$ is a reducible edge giving the reductions

$$
\left(T \circ_{k} T^{\prime}\right) \sim\left(T \circ_{k} T^{\prime}\right)_{i^{\prime} j^{\prime}} \text { and }\left(T^{\prime} \circ_{l} T\right) \sim\left(T^{\prime} \circ_{l} T\right)_{i^{\prime} j^{\prime}}
$$

The reductions are also closed under the symmetric actions: for $\sigma \in \mathbb{S}_{n}$ the edge $\overrightarrow{(i \sigma)(j \sigma)}$ is reducible in $T \sigma$. This shows the first part and in particular that $\operatorname{BCACT}_{Y}$ is an operad.

We will now show that all reductions $T \sim T_{i j}$ are obtainable from the reduction (3.2.3). We must show that any reducible $Y$-tree $T$, with reducible edge $\overrightarrow{i j}$, say, is contained in the ideal in $\operatorname{NAP}_{Y}$ generated by the left-hand side of (3.2.3). Let $\overrightarrow{k i}$ be the unique edge incoming to $i$. By applying a permutation we may assume that $k=1, i=2$ and $j=3$. The essential idea of the proof is that since the left-hand side of (3.2.3) is a subtree, the tree $T$ may be written as a composition of it and other $Y$-trees. Removing the edges $\overrightarrow{12}$ and $\overrightarrow{23}$ from $T$ leaves three connected components; $T_{1}$ contains $1, T_{2}$ contains 2 and $T_{3}$ contains 3 . In effect we have partitioned the edge set of $T$ into $\{\overrightarrow{12}, \overrightarrow{23}\}, E\left(T_{1}\right), E\left(T_{2}\right)$ and $E\left(T_{3}\right)$. Then we may express $T$ as

$$
T=\left(\left(\left(T_{1} \circ_{1}(1 \stackrel{y}{\rightarrow} 2 \stackrel{p}{\rightarrow} 3)\right) \circ_{3} T_{3}\right) \circ_{2} T_{2}\right) \cdot \sigma,
$$

where $\sigma$ is a permutation relabelling the vertices.

Remark 3.10 The Corollary 6.7 to Theorem 6.6 states that $\mathrm{NAP}_{Y}$ is binary quadratic. Along with the theorem above, this shows that $\mathrm{BCACT}_{Y}$ is also binary quadratic. 
In the spirit of how we approached $\mathrm{NAP}_{Y}$-algebras, a $\mathrm{BCACT}_{Y}$-algebra in a symmetric monoidal category $\mathcal{C}$ is a $\mathrm{NAP}_{Y}$-algebra enriched in $\mathcal{C}$ where the operation

2
$\uparrow$
$1 p$

is associative and

$$
f\left(y, \begin{array}{c}
2 \\
1_{p}
\end{array}(a, b)\right)=f(y, a) \circ f(y, b) .
$$

\subsection{The topological operad $\mathrm{BCACT}_{Y}$}

The construction $\mathrm{BCACT}_{Y}$ is functorial in the pointed set $Y$, that is, there is a functor $\mathrm{BCACT}_{(-)}$from the category of pointed sets to the category of operads. Now let $Y_{*}$ be a pointed simplicial set: this may be defined as a simplicial set $Y_{*}$ with a map from the terminal simplicial set, $\{p\}_{*}$, consisting of a singleton set in each dimension, or equivalently as a simplicial object in the category of pointed sets. By the functoriality of $\operatorname{BCACT}_{(-)}$, defining $\left(\operatorname{BCACT}_{Y}\right)_{i}=\operatorname{BCACT}_{Y_{i}}$ for $i \geq 0$, we have a simplicial operad $\operatorname{BCACT}_{Y_{*}}$. Just as a simplicial associative monoid is, equivalently, an associative monoid in the category of simplicial sets, the simplicial operad $\mathrm{BCACT}_{Y_{*}}$ can be thought of as an operad in the category of simplicial sets. Thus there is a geometric realisation of $\mathrm{BCACT}_{Y_{*}}$ as a topological operad. The same arguments apply to the operad $\mathrm{NAP}_{Y_{*}}$ for $Y_{*}$ a simplicial set.

Recall that the direct product $X_{*} \times Y_{*}$ of two simplicial sets is defined by $\left(X_{*} \times Y_{*}\right)_{i}=$ $X_{i} \times Y_{i}$ and that the geometric realisation preserves finite direct products, so $\left|X_{*} \times Y_{*}\right| \cong$ $\left|X_{*}\right| \times\left|Y_{*}\right|$. Hence geometric realisation is compatible with polynomial functors and so $\left|\mathrm{NAP}_{Y_{*}}\right| \cong \mathrm{NAP}_{\left|Y_{*}\right|}$. The face and degeneracy maps preserve the set of relations $T \sim T_{i j}$, so for a pointed simplicial set $Y_{*}$, there is a map of simplicial operads, $\mathrm{NAP}_{Y_{*}} \rightarrow \mathrm{BCACT}_{Y_{*}}$. Note that geometric realisation also preserves colimits, since it is left adjoint to the total singular complex functor. The simplicial set $\mathrm{BCACT}_{Y_{*}}$ can be written as a colimit of finite direct products of $Y_{*}$, which means that as topological operads, $\left|\mathrm{BCACT}_{Y_{*}}\right| \cong \mathrm{BCACT}_{\left|Y_{*}\right|}$.

\subsection{The fundamental groupoid of $\operatorname{BCACT}_{Y}$}

For a pointed connected simplicial set $Y_{*}$, the inclusion of simplicial sets $\{p\}_{*} \rightarrow Y_{*}$ gives inclusions $\mathrm{NAP}_{\{p\}_{*}} \rightarrow \mathrm{NAP}_{Y_{*}}$ and $\mathrm{BCACT}_{\{p\}_{*}} \rightarrow \operatorname{BCACT}_{Y_{*}}$. In the first case $\mathrm{NAP}_{\{p\}_{*}}$ is isomorphic to the discrete simplicial operad NAP. For each element $T$ 
of NAP $(n)$ there is a subsimplicial set $\mathrm{NAP}_{Y_{*}}^{T} \cong Y_{*}^{n-1}$ consisting of labellings of the rooted tree $T$. Thus the discrete simplicial operad NAP picks out a single point in each connected component of $\mathrm{NAP}_{Y}$. Using these points we may compute the fundamental groupoid $\pi_{1}\left(\left|\operatorname{NAP}_{Y_{*}}(n)\right| ; \operatorname{NAP}(n)\right)$ to be a disjoint union of groups $\left(\pi_{1}\left(\left|Y_{*}\right|, p\right)\right)^{n-1}$, one for each tree $T \in \mathrm{NAP}$.

For the cacti, the operad $\operatorname{BCACT}_{\{p\}_{*}}$ is isomorphic to the discrete simplicial operad Perm for permutative algebras. In arity $n, \operatorname{Perm}(n)$ has $n$ elements; as irreducible rooted trees these are the corollas, with every non-root vertex adjacent to the root. The simplicial set $\operatorname{BCACT}_{Y_{*}}(n)$ is the disjoint union of simplicial sets $\operatorname{BCACT}_{Y_{*}}^{v}(n)$ for $v=1, \ldots, n$, where $\mathrm{BCACT}_{Y_{*}}^{v}(n)$ consists of those rooted trees with root $v$; note that equivalences $T \sim T_{i j}$ preserve the root.

Proposition 3.11 Let $Y_{*}$ be a pointed connected simplicial set. Then the space $\operatorname{BCACT}_{Y_{*}}^{1}(n)$ is connected, and its fundamental group is presented by generators $\alpha_{i j}^{g}$ for $i=1, \ldots, n, j=2, \ldots, n$, with $i \neq j$ and $g \in \pi_{1}\left(\left|Y_{*}\right|, p\right)$, along with relations

$$
\begin{aligned}
\alpha_{i j}^{g} \alpha_{i j}^{h} & =\alpha_{i j}^{g h}, \\
{\left[\alpha_{i j}^{g}, \alpha_{i k}^{h}\right] } & =e, \\
{\left[\alpha_{i j}^{g}, \alpha_{k l}^{h}\right] } & =e, \\
{\left[\alpha_{i j}^{g} \alpha_{i k}^{g}, \alpha_{j k}^{h}\right] } & =e,
\end{aligned}
$$

for distinct $i, j, k, l$.

Proof Since $\mathrm{BCACT}_{(-)}$is a functor from pointed sets, it not only takes simplicial sets to simplicial operads, but also takes homotopies to homotopies, so it preserves homotopy equivalences. To compute the fundamental group, replace the simplicial set $Y_{*}$ with a minimal Kan subcomplex $K_{*}$ of $S\left(\left|Y_{*}\right|\right)$, the total singular complex of the geometric realisation of $Y_{*}$. For details, see May [30]: the properties we require are (a) that $K_{0}$ is a singleton set, (b) that $K_{1} \cong G:=\pi_{1}\left(\left|Y_{*}\right|\right)$ and (c) that for any pair $g, h \in G$ there exists some $\sigma_{(g, h)} \in K_{2}$ with boundary edges $(g, g h, h)$, and that, conversely, any 2 -simplex has boundary edges of this form.

Changing $Y_{*}$ to $K_{*}$ does not change the homotopy groups because the geometric realisations will be homotopic:

$$
\left|\mathrm{BCACT}_{Y_{*}}\right| \cong \mathrm{BCACT}_{\left|Y_{*}\right|} \simeq \mathrm{BCACT}_{\left|K_{*}\right|} \cong\left|\mathrm{BCACT}_{K_{*}}\right| .
$$

The isomorphisms hold because geometric realisation preserves finite products and colimits, and the middle homotopy comes from $\left|Y_{*}\right| \simeq\left|K_{*}\right|$. 
Since $K_{0}$ is a singleton set, we have $\left(\operatorname{BCACT}_{K_{*}}\right)_{0} \cong \operatorname{BCACT}_{\{p\}}$, which, as we have seen, is isomorphic to the discrete operad Perm. Hence the simplicial set $\mathrm{BCACT}_{K_{*}}^{1}(n)_{*}$ has only a single point corresponding to the corolla with root 1 and adjacent elements $2, \ldots, n$, and so it is connected.

The fundamental group of $\mathrm{BCACT}_{K_{*}}^{1}(n)_{*}$ has a group presentation with generators the set of edges $\mathrm{BCACT}_{K_{*}}^{1}(n)_{1}$ and relations given by the triangles $\operatorname{BCACT}_{K_{*}}^{1}(n)_{2}$. The set of edges consists of equivalence classes of $G$-labelled trees. Each class may be represented by a unique irreducible tree. The set of triangles consists of equivalence classes of $K_{2}$-labelled trees, and again each class may be represented by a unique irreducible tree. We will denote by $\alpha_{T}^{\left(g_{e}\right)_{e \in T}}$ the generator given by the rooted tree $T$ with the given $G$-labelling, $\left(g_{e}\right)_{e \in T}$.

Now consider a rooted tree $T$ with labelling $\left(\sigma_{e}\right)_{e \in T}$. This corresponds to a triangle in $\operatorname{BCACT}_{K_{*}}^{1}(n)_{2}$. Write $\left(g_{e}, g_{e} h_{e}, h_{e}\right)$ for the boundary edges of each $\sigma_{e}$. The relation represented by this triangle is

$$
\alpha_{T}^{\left(g_{e} h_{e}\right)_{e \in T}}=\alpha_{T}^{\left(g_{e}\right)_{e \in T}} \cdot \alpha_{T}^{\left(h_{e}\right)_{e \in T}} .
$$

Note that we did not require any of the labelled trees to be irreducible. The relations (3.4.6) along with the equivalences given by $\sim$ present the fundamental group $\pi_{1}\left(\left|\mathrm{BCACT}_{K_{*}}^{1}(n)_{*}\right|\right)$. Since for any pair of group elements $(g, h) \in G \times G$, there exists some $\sigma \in K_{2}$ with boundary edge set $(g, g h, h)$, the set of distinct relations (3.4.6) is parametrised by $G \times G$-labellings of $T$.

For distinct $i, j$ with $j \neq 1$, let

$$
T^{i j}
$$

be the tree with an edge $\overrightarrow{i j}$ and remaining edges of the form $\overrightarrow{1 m}$. If $i=1$ then $T^{i j}$ is a corolla. For distinct $i, j, k$ and $j, k \neq 1$, let

$$
\mathrm{V}^{i j k}
$$

be the tree with edges $\overrightarrow{i j}, \overrightarrow{i k}$ and remaining edges of the form $\overrightarrow{1 m}$, and let $\mathrm{I}^{i j k}$ be the tree with edges $\overrightarrow{i j}, \overrightarrow{j k}$ and remaining edges of the form $\overrightarrow{1 m}$. Again if $i=1$, then $\mathrm{V}^{i j k}$ is a corolla, but $\mathrm{I}^{i j k}=T^{j k}$. Finally, for distinct $i, j, k, l$ and $j, l \neq 1$, let $T^{i j k l}$ be the tree with edges $\overrightarrow{i j}$ and $\overrightarrow{k l}$ with remaining edges of the form $\overrightarrow{1 m}$. If $i=k=1$ then $T^{i j k l}$ is a corolla, while if $i=1, k \neq 1$, we have $T^{i j k l}=T^{k l}$.

For every tree $T$ with root 1 , the set of relations (3.4.6) of the form $\alpha_{T}^{\left(g_{e}, h_{e}\right)}$ presents the direct product of $n-1$ copies of $G$ : one copy for each edge of $T$. To identify the presentation in the statement of the proposition with $\pi_{1}\left(\left|\operatorname{BCACT}_{K_{*}}^{1}(n)_{*}\right|\right)$, we identify $\alpha_{i j}^{g}$ with $\alpha_{T^{i j}}^{\left(g_{e}^{i j}\right)}$, where $\left(g_{e}^{i j}\right)_{e \in T^{i j}}$ is the labelling that is the identity for all edges 
except $\overrightarrow{i j}$, which is labelled by $g$. We use the notation $\left(g_{e}^{i j}\right)_{e \in T}$ when we have a tree labelling with only one non-trivially labelled edge $\overrightarrow{i j}$.

The set of relations (3.4.6) coming from trees $T^{i j}$ includes the relations (3.4.1). The set of relations (3.4.6) coming from $\mathrm{V}^{i j k}$ ensure that the relations (3.4.2) are satisfied, and the tree $T^{i j k l}$ corresponds to the relations (3.4.3). Finally we look at the trees $\mathrm{I}^{i j k}$ : the $G \times G$-labellings ensure that commutation relations are satisfied, ie

$$
\left[\alpha_{\mathrm{I}^{i j k}}^{\left(g_{e}^{i j}\right)}, \alpha_{\mathrm{I}^{i j k}}^{\left(h_{e}^{j k}\right)}\right]
$$

If $i=1$, then $\alpha_{\mathrm{I}^{i j k}}^{\left(h_{e}^{j k}\right)}$ is equal to $\alpha_{j k}^{h}$. If $i \neq 1$, then it is reducible along the edge $\overrightarrow{i j}$; the reduction removes this edge and replaces it with $\overrightarrow{1 j}$, labelled with the identity, so one still gets $\alpha_{j k}^{h}$. Whether $i$ is equal to 1 or not, the element

$$
\alpha_{\mathrm{I}^{i j k}}^{\left(g_{e}^{i j}\right)}
$$

is reducible along the edge $\overrightarrow{j k}$. When reducing, the edge $\overrightarrow{j k}$ is removed and replaced by $\overrightarrow{i k}$, leaving the tree $V^{i j k}$. However in this case the edge $\overrightarrow{i k}$ inherits the label $g$ from $\overrightarrow{i j}$. But recall that $\mathrm{V}^{i j k}$ corresponds to a direct product, allowing us to write this reduced tree as the product of two trees, each with a single label of $g$ :

$$
\alpha_{\mathrm{I}^{i j k}}^{\left(g_{e}^{i j}\right)}=\alpha_{\mathrm{V}^{i j k}}^{\left(g_{e}^{i j}\right)} \cdot \alpha_{\mathrm{V}^{i j k}}^{\left(g_{e}^{i k}\right)}
$$

But these last two elements have reducible edges, $\overrightarrow{i k}$ and $\overrightarrow{i j}$, respectively; reducing along these, we have

$$
\alpha_{\mathrm{I} i k}^{\left(g_{e}^{i j}\right)}=\alpha_{i j}^{g} \cdot \alpha_{i k}^{g}
$$

meaning that (3.4.7) becomes the relation (3.4.4).

This accounts for all the generators and relations in the smaller presentation. We now show that with the exception of the trees

$$
T^{i j}, \quad \mathrm{~V}^{i j k}, \quad \mathrm{I}^{i j k} \text { and } T^{i j k l} \text {, }
$$

the generators and relations (3.4.6) associated to a tree $T$ may be written in terms of the smaller presentation; they are not needed. In fact we show that for a tree $T$ with three of more edges not emanating from the root 1 , the generators $\alpha_{T}^{\left(g_{e}\right)}$ can be rewritten as trees of strictly lower level, and the relations $\alpha_{T}^{\left(g_{e}, h_{e}\right)}$ may be rewritten in terms of relations of lower level. This will complete the proof.

So let $T$ be such a tree, with edges $\overrightarrow{i j}, \overrightarrow{k l}$ and $\overrightarrow{p q}$ for which $i, k, p \neq 1$. The generators and relations associated to labellings of $T$ present a direct product of $n-1$ 
copies of $G$. To start, we replace this presentation with the subset of generators with labellings that are only non-trivial on one edge, ie the factors of the direct product. We choose a subset of relations consisting of $G \times G$-labellings of $T$ that are non-trivial on no more than two edges; this includes the relations in individual factors and the commutation relations between pairs of factors. These generators and relations present the same direct product. Now given any such generator or relation, one of the three edges $\overrightarrow{i j}, \overrightarrow{k l}, \overrightarrow{p q}$ must be trivially labelled; let us choose $\overrightarrow{i j}$. Reducing along this edge we get the reduced tree $T_{i j}$, which has strictly lower level, so if we started with a generator we have written the generator in terms of lower level trees, and if we start with a relation, this relation still holds in the direct product associated to $T_{i j}$.

Since $\mathrm{BCACT}_{Y_{*}}$ is a simplicial operad with a suboperad $\mathrm{BCACT}_{\{p\}_{*}}$ of points, the fundamental groupoid $\pi_{1}\left(\left|\mathrm{BCACT} Y_{*}\right|, \mathrm{BCACT}_{\{p\}_{*}}\right)$ is an operad in groupoids. To give the composition maps, we need only describe the compositions on the generating morphisms. In fact since we have $g \circ_{i} h=\left(g \circ_{i} e\right) \cdot\left(e \circ_{i} h\right)$ we need only describe the compositions of generators with identity maps.

Proposition 3.12 Let $\left(Y_{*}, p\right)$ be a connected pointed simplicial set and let $G$ be its fundamental group. The operad structure on $\pi_{1}\left(\left|\mathrm{BCACT}_{Y_{*}}\right|, \mathrm{BCACT}_{\{p\}_{*}}\right)$ is given on generating morphisms as follows: let $\alpha_{i j}^{g} \in \operatorname{BCACT}_{G}(n)_{r}$ and $e \in \operatorname{BCACT}_{G}(m)_{s}$ be the identity morphism. For $a \in[m]$, define $i^{\prime}=i+a-1$ and $j^{\prime}=j+a-1$; then we have

$$
e \circ_{a} \alpha_{i j}^{g}=\alpha_{i^{\prime} j^{\prime}}^{g} .
$$

For $b \in[n]$, define $i^{\prime \prime}$ to be $i$ if $i<b$, to be $i+m-1$ if $i>b$ and $i+s-1$ if $i=b$; define $j^{\prime \prime}$ similarly. For each $l \in[m]$ define $l^{\prime \prime}$ to be $l+b-1$. Then we have

$$
\alpha_{i j}^{g} \circ_{b} e= \begin{cases}\prod_{l=1}^{m} \alpha_{i^{\prime \prime} l^{\prime \prime}}^{g} & \text { if } b=j, \\ \alpha_{i^{\prime \prime} j^{\prime \prime}}^{g} & \text { otherwise. }\end{cases}
$$

Proof Let $T_{r}^{i j}$ be the tree with root $r$, the edge $\overrightarrow{i j}$ and $(n-2)$ edges $\overrightarrow{r k}$ (if $i=r$ then this is a corolla). Then $\alpha_{i j}^{g}$ is represented by the tree

$$
T_{r}^{i j}
$$

with $\overrightarrow{i j}$ labelled by $g \in G$. Let $C_{s}$ be the corolla with root $s$ and $m-1$ edges $\overrightarrow{s k}$. When all of the edges are labelled by the identity $e \in G$ then this represents the identity $e$ of $\mathrm{BCACT}_{G}(m)_{s}$. 
To compute $e \circ_{a} \alpha_{i j}^{g}$ we compose trees to get $C_{s} \circ_{a} T_{r}^{i j}$ and then reduce as follows. The unique labelled edge $\overrightarrow{i j}$ of $T_{r}^{i j}$ is a leaf and hence it is also a leaf of

$$
C_{s} \circ_{a} T_{r}^{i j}
$$

although now the edge is $\overrightarrow{i^{\prime} j^{\prime}}$. Since it is a leaf it reduces to $\alpha_{i^{\prime} j^{\prime}}^{g}$ as required.

To compute $\alpha^{g}{ }_{o_{b}} e$ is a little more complicated as it depends on the value of $b$. If $b \neq j$ then the leaf

$$
\overrightarrow{i j}
$$

is still a leaf of $T_{r}^{i j} \circ_{b} C_{s}$ and so the same argument applies to give the reduction to $\alpha_{i^{\prime \prime} j^{\prime \prime}}^{g}$. However if $b=j$ then the tree consists of the edge

$$
\overrightarrow{i^{\prime \prime} j^{\prime \prime}}
$$

another $n-2$ edges emanating from the root and $m-1$ edges $\overrightarrow{j^{\prime \prime} l^{\prime \prime}}$. The only labelled edge is

$$
\overrightarrow{i^{\prime \prime} j^{\prime \prime}}
$$

and the set $A_{i j}$ of vertices "above $i$ " consists of the vertex $j^{\prime \prime}=s^{\prime \prime}$ and the vertices $l^{\prime \prime}$ for each edge $\overrightarrow{j l} \in C_{s}$. Repeated reductions yield a tree $T$ with edges

$$
\overrightarrow{i^{\prime \prime} l^{\prime \prime}}
$$

for $l=1, \ldots, m$ labelled by $g$ and all other edges joined to the root. Now this element may be rewritten as a product of $n$ elements corresponding to labellings of $T$ with a single edge

$$
\overrightarrow{i^{\prime \prime} l^{\prime \prime}}
$$

labelled by $g$. Reducing each of these elements gives a generator $\alpha_{i^{\prime \prime} l^{\prime \prime}}^{g}$ and hence the required result.

Remark 3.13 The groups $\operatorname{BCACT}_{G}(n)_{r}$ act faithfully on the free product $G^{* n}$. We will write this free product as $G_{1} * \cdots * G_{n}$ where each group is isomorphic to $G$ in order to distinguish between different factors. The element $\alpha_{i j}^{g}$ acts on the factors as follows:

$$
\alpha_{i j}^{g}(h)= \begin{cases}h^{g_{i}^{-1}} & \text { if } h \in G_{j} \text { and where } g_{i}=g \text { in } G_{i}, \\ h & \text { if } h \in G_{k} \text { for } k \neq j .\end{cases}
$$

In [18] the closely related spaces of unbased cacti CACTY were studied, and it was shown that when $Y_{i}$ is a classifying space for $G_{i}$ then $\mathrm{CACT}_{\mathbf{Y}}$ is itself a classifying 
space for a certain group of automorphisms. As a consequence of Theorems 4.6 and 6.12, we see that

$$
H_{*}\left(\mathrm{BCACT}_{Y_{*}}\right) \cong \operatorname{Perm} \circ \mathrm{NAP} \bar{H}_{*}(Y),
$$

whereas in [18] it is shown that

$$
H_{*}\left(\mathrm{CACT}_{Y_{*}}\right) \cong \operatorname{Com} \circ \mathrm{NAP}_{\bar{H}_{*}(Y)} .
$$

This last isomorphism could also be shown using the methods of reduction used in this paper, although $\mathrm{CACT}_{Y_{*}}$ is not an operad.

\subsection{Relationships with other topological operads}

The pure braid group on $n$ strands, $P_{n}$, is known to be a subgroup of the group $P \Sigma_{n} \cong \pi_{1}\left(\mathrm{CACT}_{S^{1}}(n)\right)$ of partial conjugations of the free group on $n$ letters. This inclusion may be realised by a construction involving cacti. In Kaufmann [20] various (quasi-)operads of cacti are discussed; these are different from the operad $\mathrm{BCACT}_{S^{1}}$ in that the cacti are planar and unbased. We will take PlCACT to be the spineless and normalised varieties of cacti from [20]. This quasi-operad is quasi-isomorphic to the little discs operad, and so in particular the fundamental group $\pi_{1}(\operatorname{PlCACT}(n))$ is the pure braid group $P_{n}$. There is an $\mathbb{S}_{n}$-equivariant map

$$
\operatorname{PlCACT}(n) \rightarrow \operatorname{CACT}_{S^{1}}(n)
$$

defined by the map that forgets the planar structure of a planar cactus, leaving a cactus product of circles as defined in (3.2.1); on fundamental groups this gives the inclusion $P_{n} \rightarrow P \Sigma_{n}$. The operad compositions of $\mathrm{BCACT}_{S^{1}}$ and PlCACT are not closely related; this may be seen by examining the homology operads that are $\operatorname{BCACT}_{H_{*}}\left(S^{1}\right)$, as defined in the next section and the Gerstenhaber operad $e_{2}$.

However both families of cacti are related by a third operad, which "contains" both. Let $\operatorname{LR}(n)$ be the space of smooth, disjoint embeddings of $n$ copies of the filled in torus, or ring $R=S^{1} \times D^{2}$ into itself - this is naturally an operad. The little discs operad consists of disjoint embeddings of copies of a disc $D^{2}$ into itself and can be mapped into the little rings operad LR by applying $\operatorname{id}_{S^{1}} \times(-)$ to the embeddings. The image of the little discs operad involves little rings which wind around the large ring once. Meanwhile the operad $\mathrm{BCACT}_{S^{1}}$ is related to the connected components of embeddings in which one little ring, the root, winds around the large ring once; the remaining rings do not wind around the large ring and all of the rings are unknotted and unlinked. The fundamental groups of these connected components contain $\pi_{1}\left(\mathrm{BCACT}_{S^{1}}\right) \cong \mathrm{BCACT}_{\mathbb{Z}}$ as a suboperad. There are additional elements not in the suboperad given by little rings circling through the large ring along with smooth endomorphisms of $R$. 


\section{The homology operads}

So far we have described operads $\operatorname{NAP}_{Y}$ and $\mathrm{BCACT}_{Y}$ in the "geometric" setting. Both families also have versions existing in the "linear" setting, so for any graded vector space $D$ there exists an operad $\mathrm{NAP}_{D}$, whereas in the case of the based cacti there is a subtlety: we require a graded augmented cocommutative coalgebra $C$ to define $\mathrm{BCACT}_{C}$. The "geometric" and "linear" versions are closely related via the homology functor, which sends a simplicial set to its homology groups with coefficients in the base field $\mathbb{k}$. In this section, we shall describe these operads via constructions with decorated rooted trees, and later in Section 6, we shall describe them via generators and relations, and show that in fact each of them has a quadratic Gröbner basis of relations.

\subsection{The linear operad NAP}

Let $D$ be a graded vector space (over some field $\mathbb{k}$ ). Recall that in (3.1.1) we described $\operatorname{NAP}_{Y}(n)$ as disjoint union of direct products of copies of $Y$. Then in Remark 3.2 we gave a map of sets (3.1.10) realising $\operatorname{NAP}_{Y}(n)$ as a polynomial functor in $Y$. Let $D$ be a graded vector space and define $\mathrm{NAP}_{D}$ via the same polynomial functor in the category of graded vector spaces:

$$
\operatorname{NAP}_{D}(n)=\bigoplus_{T \in \operatorname{RT}(n)} D^{\otimes(n-1)} .
$$

Equivalently, $\operatorname{NAP}_{D}(n)$ is the vector space spanned by rooted trees with vertex set $[n]$ and edge labels in $D$, subject to linearity in each edge label.

The set based description of the $\operatorname{NAP}_{Y}$ operad works on the level of polynomial functors and so suffices to show that $\mathrm{NAP}_{D}$ is an operad. However great care must be taken to keep track of the signs induced by the symmetry $\sigma$ from the symmetric monoidal category ( $\mathrm{gVect}, \otimes, \sigma, \mathbb{k}$ ) of graded vector spaces. In order to do this we must assign for each term $D^{\otimes n-1}$ in the sum (4.1.1) a reference ordering of the factors. This requires assigning to each tree $T \in \mathrm{RT}(n)$ a total ordering on the set of edges $E(T)$. Let $T$ be such a tree and let $i$ be its root. Since each vertex has a unique incoming edge except for the root which has none, the set of edges $E(T)$ is in bijection with the set of non-root vertices $[n]-i$. We take the ordering of $E(T)$ from the natural ordering of $[n]-i$. So for instance the pair

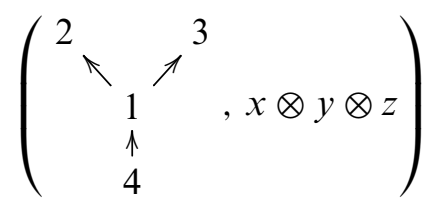

represents the $Y$-tree

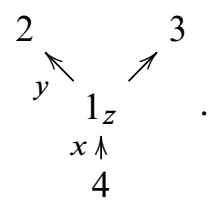


The order of $x, y$ and $z$ in the tensor product is determined by the order of the edges. The first step in giving the operad structure is to describe the action of the symmetric group $\mathbb{S}_{n}$ on $\operatorname{NAP}_{D}(n)$. For instance applying the permutation (24) to the $Y$-tree considered in (4.1.2), we get:

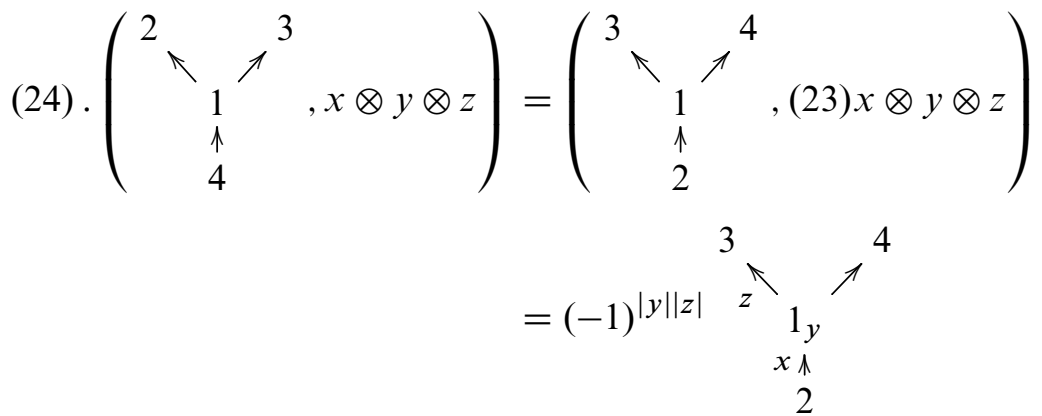

The signs involved in the composition $T \circ_{i} T^{\prime}$ for $T \in \operatorname{NAP}_{D}(n)$ and $T^{\prime} \in \operatorname{NAP}_{D}(m)$ are more easily accounted for. This is because the edges within the right-hand tree $T^{\prime}$ are not reordered within $T \circ_{i} T^{\prime}$ and so the sign depends on the total degree $\left|T^{\prime}\right|$ and not on the individual edges. The edges of $T^{\prime}$ are "moved past" the edges $\overrightarrow{j k} \in E(T)$ for which $k>i$. Hence if $y_{j k}$ is the labelling of $\overrightarrow{j k}$, the sign change is given by

$$
(-1)^{\left|T^{\prime}\right|\left(\sum_{\overrightarrow{j k} \in E(T) \mid k>i}\left|y_{j k}\right|\right)} \text {. }
$$

Proposition 4.1 The homology operad $H_{*}\left(\mathrm{NAP}_{Y_{*}}\right)$ with coefficients in the base field $\mathbb{k}$ is isomorphic to the linear operad $\operatorname{NAP}_{H_{*}(Y)}$.

Proof With field coefficients, the homology functor $H_{*}$ from simplicial sets to graded vector spaces sends direct products to tensor products and preserves coproducts, and so is compatible with polynomial functors. The explicit expression of this is

$$
H_{*}\left(\operatorname{NAP}_{Y}(n)\right) \cong H_{*}\left(\coprod_{T \in \operatorname{RT}(n)} Y^{E(T)}\right) \cong \bigoplus_{T \in \operatorname{RT}(n)} H_{*}(Y)^{\otimes E(T)}
$$

\subsection{The linear operads of based cacti}

Let $C$ be an augmented cocommutative coalgebra and write its splitting as $\mathbb{1} \oplus \bar{C}$. The operad $\mathrm{BCACT}_{C}$ will be a quotient of the operad $\mathrm{NAP}_{C}$; this is a parallel of the set-based versions. Let $T \in \mathrm{NAP}_{C}$ be a $C$-labelled rooted tree and suppose that it has an edge $\overrightarrow{i j}$ with the label $\mathbb{1}$, and suppose further that $i$ is not the root of $T$; as before, we will call the edge $\overrightarrow{i j}$ reducible. Let $k$ be the unique vertex such that $\overrightarrow{k i}$ is an edge and let $c$ be the label of $\overrightarrow{k i}$. We define $T^{\prime}$ to be the unlabelled rooted tree created by 
removing the edge $\overrightarrow{i j}$ and replacing it by $\overrightarrow{k j}$, and denote by $T^{\prime}(a, b)$ the edge labelled rooted tree based on $T^{\prime}$ where the edge labels are inherited from those of $T$ except for $\overrightarrow{k i}$, which is labelled by $a$, and $\overrightarrow{k j}$, which is labelled by $b$. Finally we define $T_{i j}$ to be the sum

$$
\sum(-1)^{\left|c_{(2)}\right| g} T^{\prime}\left(c_{(1)}, c_{(2)}\right)
$$

where $g$ is the sum of degrees

$$
\sum_{\overrightarrow{x y} \mid i<y<j}\left|a_{x y}\right|
$$

and $a_{x y}$ is the label of the edge $\overrightarrow{x y}$. The sign is given by the moving of the label $c_{(2)}$ from being adjacent to $c_{(1)}$ as in $\Delta(c)=\sum c_{(1)} \otimes c_{(2)}$ to being in the relevant position to label the edge $\overrightarrow{k j}$. As before $T_{i j}$ is called the reduction of $T$ at the reducible edge $\overrightarrow{i j}$ and just as before each $C$-tree reduces to a unique irreducible $C$-tree.

Definition 4.2 The graded vector space of linear based $C$-cacti, BCACT $C$, is defined by factoring out from $\mathrm{NAP}_{C}$ the relations

$$
T-T_{i j}=0
$$

for trees $T$ with an edge $\overrightarrow{i j}$ labelled by $\mathbb{1}$ where $i$ is not the root.

The graded vector space of irreducible $C$-trees and hence $\mathrm{BCACT}_{C}$ is given by

$$
\operatorname{BCACT}_{C}(n) \cong \bigoplus_{T \in \mathrm{RT}(n)}\left(\bigotimes _ { r ( T ) j \in E ( T ) } \left(\bigotimes_{\substack{i j \in E(T), i \neq r(T)}} C\left(\bigotimes_{i}\right),\right.\right.
$$

where $r(T)$ is the root of $T$. Using the splitting $C=\mathbb{k} \mathbb{1} \oplus \bar{C}$, we may rewrite this as a polynomial expression in $\bar{C}$. There is a convenient way of indexing this polynomial; rather than using irreducible $C$-trees, where an outgoing edge $\overrightarrow{r j}$ from the root $r$ could be labelled by $\mathbb{1}$, we cut the edges $\overrightarrow{r j}$ labelled by $\mathbb{1}$ to leave a labelled forest, each component tree has a root, the corresponding $j$, and there is a chosen component tree, the tree containing $r$. Let $\mathrm{PF}_{*}$ be the set of planted forests with a chosen tree. Then we may rewrite (4.2.4) as

$$
\operatorname{BCACT}_{C}(n) \cong \bigoplus_{F \in \mathrm{PF}_{*}(n)} \bar{C}^{\otimes E(F)} .
$$

Remark 4.3 Although this is a polynomial functor in $\bar{C}$ with a similar diagram to (3.1.10), the operad maps are not maps of polynomials: indeed, the diagonal map of $C$ 
is used. A similar polynomial description of $\operatorname{BCACT}_{Y}$ holds when $Y$ is a set; however, this involves "splitting" the chosen point of $Y$ and so this only works for a pointed simplicial set when the point is disconnected.

Proposition 4.4 The linear subspace of $\mathrm{NAP}_{C}$ generated by relations of the form $T-T_{i j}=0$ is an operadic ideal and so $\mathrm{BCACT}_{C}$ is an operad as a quotient of $\mathrm{NAP}_{C}$. Furthermore the ideal is generated in arity 3 by

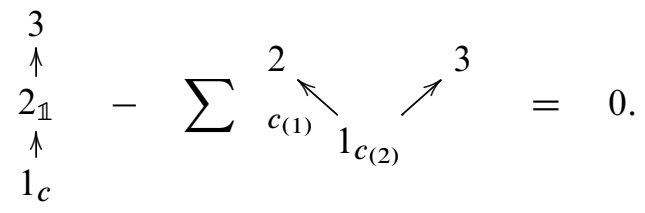

Proof This is the linear analogue of Theorem 3.9, and the same method applies.

Example 4.5 Let us give explicit presentations for the operads $\mathrm{BCACT}_{C}$ for the two simplest non-trivial choices of $C$. For the split two-dimensional coalgebra $\mathbb{k} \oplus \mathbb{k}$, we denote by $\cdot_{0}$ and $\cdot_{1}$ its elements corresponding to the two points of $Y$, and observe that the following identities hold in every algebra over $\mathrm{BCACT}_{\mathbb{k} \oplus \mathbb{k}}$ :

$$
\begin{aligned}
& \left(a \cdot \cdot_{0} b\right) \cdot \cdot_{0} c=a \cdot_{0}\left(b \cdot \cdot_{0} c\right), \\
& \left(a \cdot \cdot_{0} b\right) \cdot \cdot_{0} c=(-1)^{|b||c|}(a \cdot 0 c) \cdot \cdot_{0} b, \\
& \left(a \cdot \cdot_{0} b\right) \cdot{ }_{1} c=(-1)^{|b||c|}\left(a \cdot \cdot_{1} c\right) \cdot \cdot_{0} b, \\
& \left(a \cdot \cdot_{1} b\right) \cdot \cdot_{1} c=(-1)^{|b||c|}\left(a \cdot \cdot_{1} c\right) \cdot \cdot_{1} b, \\
& a \cdot \cdot_{1}\left(b \cdot \cdot_{0} c\right)=\left(a \cdot \cdot_{0} b\right) \cdot{ }_{0} c .
\end{aligned}
$$

For the exterior coalgebra $\Lambda_{\mathbb{k}}\langle x\rangle$ of a one-dimensional space, we denote by $\cdot$ and $\bullet$ its elements corresponding to $\mathbb{1}$ and to the primitive element $x$ respectively, and observe that the following identities hold in every algebra over $\operatorname{BCACT}_{\Lambda_{\mathbb{R}}\langle x\rangle}$ :

$$
\begin{aligned}
(a \cdot b) \cdot c & =a \cdot(b \cdot c), \\
(a \cdot b) \cdot c & =(-1)^{|b||c|}(a \cdot c) \cdot b, \\
(a \cdot b) \bullet c & =(-1)^{|b||c|}(a \bullet c) \cdot b, \\
(a \bullet b) \bullet c & =(-1)^{1+|b||c|}(a \bullet c) \bullet b, \\
a \bullet(b \cdot c) & =(a \cdot b) \bullet c+(a \bullet b) \cdot c .
\end{aligned}
$$

Theorem 4.6 For $Y_{*}$ a pointed simplicial set, the homology operad of $\mathrm{BCACT}_{Y_{*}}$ is isomorphic to the linear operad $\mathrm{BCACT}_{H_{*}(Y)}$. 
Proof The homology functor respects products and coproducts and hence polynomial functors; this is how we see that $H_{*}\left(\mathrm{NAP}_{Y_{*}}\right) \cong \mathrm{NAP}_{H_{*}(Y)}$. However, the cactus operad $\mathrm{BCACT}_{Y_{*}}$ is not given by a polynomial functor. We have also seen that the process of reduction can be applied to $\mathrm{BCACT}_{C}$ for $C$ a graded coaugmented commutative coalgebra. One might hope to apply the chain complex functor $C_{*}$ to $Y_{*}$; however, $C_{*}$ is not monoidal and so $C_{*}(Y)$ is not necessarily a coalgebra. Instead we proceed via simplicial vector spaces.

The vector space with basis $Y_{*}$ is a simplicial object $\mathbb{k} Y_{*}$ in the category of vector spaces. The functor from simplicial sets to simplicial vector spaces is monoidal, where the tensor product of simplicial vector spaces $A_{*}$ and $B_{*}$ is given by $\left(A_{*} \otimes B_{*}\right)_{i}=A_{i} \otimes B_{i}$. Hence $\mathbb{k} Y_{*}$ is a coaugmented cocommutative coalgebra and splits as $\mathbb{k}_{*} \oplus \overline{\mathbb{k} Y_{*}}$, where $\mathbb{k}_{*}$ is a one-dimensional vector space in each degree. Thus we may form the vector space of $\mathbb{k} Y_{*}$-labelled rooted trees and say that such a tree $T$ is reducible if an edge $\overrightarrow{i j}$, for $i$ not the root, is labelled by an element of $k \mathbb{1}_{*}$. By using the coproduct, we may define the reduced tree $T_{i j}$ and by adding in the relation $T \sim T_{i j}$ we may form the space $\mathrm{BCACT}_{\mathbb{k}} Y_{*}$ of $\mathbb{k} Y_{*}$-labelled trees. This is isomorphic to $\mathbb{k B C A C T}_{Y_{*}}$; all the relations are just the linearisation of the setwise reductions $T \sim T_{i j}$. However in the linear world there is the splitting $\mathbb{k} \mathbb{1} \oplus \overline{\mathbb{k} Y_{*}}$ and so $\mathbb{k B C A C T}_{Y_{*}}(n)$ splits as

$$
\bigoplus_{T \in \operatorname{RT}(n)}\left(\underset{r(T) j \in E(T)}{\left.\bigotimes_{r(j)} \mathbb{k} Y_{*}\right) \otimes(} \underset{\overrightarrow{i j \in E(T), i \neq r(T)}}{\bigotimes} \overline{\mathbb{k} Y_{*}}\right) .
$$

Taking the homology we now have a splitting with terms

$$
\left.H_{*}(\underbrace{}_{\overrightarrow{r(T) j \in E(T)}} \mathbb{k} Y_{*}) \otimes\left(\bigotimes_{\substack{i j \in E(T), i \neq r(T)}}^{\bigotimes \bar{k} Y_{*}}\right)\right) .
$$

Since we are working over a field $\mathbb{k}$ we may apply the Künneth formula to find

$$
H_{*}\left(\operatorname{BCACT}_{Y_{*}}(n)\right)
$$

This is isomorphic to $\mathrm{BCACT}_{H_{*}(Y)}$ as a vector space. That these are isomorphic as operads is immediate because both cacti operads are defined as quotients of NAP operads.

Remark 4.7 (1) In the linear setting, the formula (3.1.13) (and its particular case (3.1.14)), as well as (3.1.15), work without any changes (except for signs that one 
should carefully trace), while the formula (3.2.7) should be adapted into

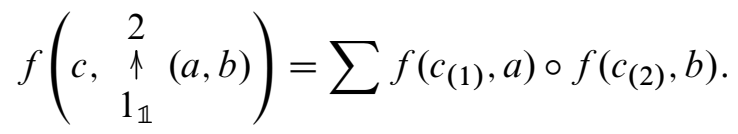

(2) Since, when $C=H_{*}(Y)$, the operad BCACT $C$ is the homology operad of a topological operad, it should not be surprising at all that for every coalgebra $C$ the operad $\mathrm{BCACT}_{C}$ is a Hopf operad (Getzler and Jones [16], and Moerdijk [32]), which essentially means that algebras over it form a tensor category. Its diagonal map coincides with the diagonal of the coalgebra $C$ on the space of generators:

$$
\Delta\left(\begin{array}{c}
2 \\
\uparrow \\
1_{c}
\end{array}\right)=\sum \begin{gathered}
2 \\
\uparrow \\
1_{c_{(1)}}
\end{gathered} \otimes \begin{gathered}
2 \\
\uparrow \\
1_{c_{(2)}}
\end{gathered}
$$

Let us conclude this section with an example of a "smallest nontrivial algebra" over a linear operad of based cacti.

Example 4.8 Let $Y$ be the (pointed) two-element set $\{0,1\}$ so that $C=H_{*}(Y)$ is the split two-dimensional coalgebra $\mathbb{k} \oplus \mathbb{k}$. As we know from Example 4.5, the product ${ }_{0} 0$ defines a Perm-algebra, and the product $\cdot{ }_{1}$ defines an NAP-algebra, and there are compatibility relations between them. In every one-dimensional $\mathrm{BCACT}_{C}-$ algebra, the Perm-product is commutative, and the NAP-product is associative, so they are very degenerate, and the first nontrivial example should be at least two-dimensional. One can easily check that a two-dimensional noncommutative Perm-algebra is necessarily isomorphic to the algebra $A=\{a, b\}$ with multiplication table

$$
\begin{aligned}
& a \cdot{ }_{0} a=a, \\
& a \cdot \cdot_{0} b=b \cdot{ }_{0} b=0, \\
& b \cdot{ }_{0} a=b .
\end{aligned}
$$

Furthermore, to define a $\mathrm{BCACT}_{C}$-algebra structure on $A$, one should choose a $2 \times 2-$ matrix $p$ with $p^{2}=p$, and put

$$
\begin{aligned}
& a \cdot \cdot_{1} a=p_{11} a+p_{12} b, \\
& b \cdot \cdot_{1} a=p_{21} a+p_{22} b, \\
& a \cdot{ }_{1} b=b \cdot{ }_{1} b=0 .
\end{aligned}
$$


One particular example will be obtained if we put $p=\left(\begin{array}{ll}0 & 0 \\ 0 & 1\end{array}\right)$, so that the NAP-product in this algebra is given by

$$
\begin{aligned}
& a \cdot \cdot_{1} a=a \cdot \cdot_{1} b=b \cdot{ }_{1} b=0, \\
& b \cdot \cdot_{1} a=b .
\end{aligned}
$$

This product is "nontrivial" enough: it has a noncommutative Perm-product, a nonassociative NAP-product, and moreover it does not fit into the series of algebras defined in Example 3.4 (since we have $a \cdot 1 a=0$ but $b \cdot 1 a=b \neq 0$ ).

\section{Filtered distributive laws}

\subsection{Filtered distributive laws between quadratic operads}

Assume that $\mathscr{A}=\mathscr{F}(\mathscr{V}) /(\mathscr{R})$ and $\mathscr{B}=\mathscr{F}(\mathscr{W}) /(\mathscr{S})$ are two quadratic operads. For two subcollections $\mathscr{U}_{1}$ and $\mathscr{U}_{2}$ of the same operad $\mathscr{O}$, let us denote by $\mathscr{U}_{1} \bullet \mathscr{U}_{2}$ the subcollection of $\mathscr{O}$ spanned by all elements $\phi \circ_{i} \psi$ with $\phi \in \mathscr{U}_{1}, \psi \in \mathscr{U}_{2}$. We shall now describe a way to define quotients of $\mathscr{F}(\mathscr{V} \oplus \mathscr{W})$ of a particular type. For any $\mathbb{S}$-module mappings

$$
\begin{aligned}
& s: \mathscr{R} \rightarrow \mathscr{W} \bullet \mathscr{V} \oplus \mathscr{V} \bullet \mathscr{W} \oplus \mathscr{W} \bullet \mathscr{W}, \\
& d: \mathscr{W} \bullet \mathscr{V} \rightarrow \mathscr{V} \bullet \mathscr{W} \oplus \mathscr{W} \bullet \mathscr{W},
\end{aligned}
$$

one can define a quadratic operad $\mathscr{E}$ with generators $\mathscr{U}=\mathscr{V} \oplus \mathscr{W}$ and relations $\mathscr{T}=\mathscr{Q} \oplus \mathscr{D} \oplus \mathscr{S}$, where

$$
\mathscr{Q}=\{x-s(x) \mid x \in \mathscr{R}\}, \quad \mathscr{D}=\{x-d(x) \mid x \in \mathscr{W} \bullet \mathscr{V}\} .
$$

Informally, we join generators of $\mathscr{A}$ and $\mathscr{B}$ together, keep the relations of $\mathscr{B}$, deform relations of $\mathscr{A}$, adding to them "lower terms" of degree at most 1 in generators of $\mathscr{A}$, and impose a rewriting rule transforming $\mathscr{W} \bullet \mathscr{V}$ into a combination of terms from $\mathscr{V} \bullet \mathscr{W}$ and "lower terms" of degree 0 in generators of $\mathscr{A}$. Note that using the rewriting rule $x \mapsto d(x)$, one can replace $s$ by

$$
s^{\prime}: \mathscr{R} \rightarrow \mathscr{V} \bullet \mathscr{W} \oplus \mathscr{W} \bullet \mathscr{W},
$$

and from now on we shall denote by $s$ that modified mapping.

Assume that the natural projection of $\mathbb{S}$-modules $\pi: \mathscr{E} \rightarrow \mathscr{A}$ splits (for example, it is always true in characteristic zero, or in arbitrary characteristic whenever the relations of $\mathscr{A}$ remain undeformed, including the case of usual distributive laws). Then the composite of natural mappings

$$
\mathscr{F}(\mathscr{V}) \circ \mathscr{F}(\mathscr{W}) \hookrightarrow \mathscr{F}(\mathscr{V} \oplus \mathscr{W}) \rightarrow \mathscr{F}(\mathscr{V} \oplus \mathscr{W}) /(\mathscr{T})
$$


gives rise to a surjection of $\mathbb{S}$-modules

$$
\xi: \mathscr{A} \circ \mathscr{B} \rightarrow \mathscr{E}
$$

Definition 5.1 We say that the mappings $s$ and $d$ above define a filtered distributive law between the operads $\mathscr{A}$ and $\mathscr{B}$ if $\pi: \mathscr{E} \rightarrow \mathscr{A}$ splits, and the restriction of $\xi$ to weight- 3 elements

$$
\xi_{3}:(\mathscr{A} \circ \mathscr{B})_{(3)} \rightarrow \mathscr{E}_{(3)}
$$

is an isomorphism.

The following result (generalising the distributive law criterion for operads that was first stated in Markl [29]) was proved in Dotsenko [10] using the set operad filtration method of Khoroshkin [21], and in Vallette [35] using a filtration on the Koszul complex; however, both proofs rely on the Künneth formula for symmetric collections and thus are not available in positive characteristic because in that case the group algebras $\mathbb{k} \mathbb{S}_{n}$ are not semisimple.

Theorem 5.2 Assume that the operads $\mathscr{A}$ and $\mathscr{B}$ are Koszul, and that the mappings $s$ and $d$ define a filtered distributive law between them. Then the operad $\mathscr{E}$ is Koszul, and the $\mathbb{S}$-modules $\mathscr{A} \circ \mathscr{B}$ and $\mathscr{E}$ are isomorphic.

Proof Let us first note that either of the characteristic zero proofs mentioned above (set operad filtration, filtration on the Koszul complex) works in the category of shuffle operads for arbitrary characteristic, since Künneth formula over a field is always available. Also, a symmetric operad $\mathscr{O}$ is Koszul if and only if it is Koszul as a shuffle operad, which proves the first statement of the theorem. To prove the second statement, we observe that in the category of nonsymmetric collections we have an isomorphism $\mathscr{E}^{f} \simeq \mathscr{A}^{f} \circ_{\mathrm{sh}} \mathscr{B}^{f} \simeq(\mathscr{A} \circ \mathscr{B})^{f}$, and in the symmetric category we have a surjection $\mathscr{A} \circ \mathscr{B} \rightarrow \mathscr{E}$. Since the forgetful functor from the category of symmetric collections to the category of nonsymmetric collections is one-to-one on isomorphism classes of objects, that surjection has to be an isomorphism.

Example 5.3 The following filtered distributive law was discussed by the first author in [10], as related to Gelfand-Varchenko algebras of locally constant functions on the complement to a hyperplane arrangement; unlike all other results of this paper, it is only available in characteristic zero. It is well-known that the associative operad admits an alternative description as an operad generated by a symmetric binary operation $\cdot \star$ • 
and a skew-symmetric binary operation $[\cdot, \cdot]$ that satisfy the relations

$$
\begin{gathered}
{[a,[b, c]]+[b,[c, a]]+[c,[a, b]]=0,} \\
{[a \star b, c]=a \star[b, c]+[a, c] \star b,} \\
(a \star b) \star c-a \star(b \star c)=[b,[a, c]] .
\end{gathered}
$$

If we put $\mathscr{V}=\operatorname{span}(\cdot \star \cdot), \mathscr{W}=\operatorname{span}([\cdot, \cdot])$, and consider the operads $\mathscr{A}=$ Com and $\mathscr{B}=$ Lie,

$$
\begin{gathered}
s((a \star b) \star c-a \star(b \star c))=[b,[a, c]], \\
d([a \star b, c])=a \star[b, c]+[a, c] \star b,
\end{gathered}
$$

then there are no additional relations in weight 3 , and in characteristic zero the projection As $\rightarrow$ Com splits, therefore the associative operad is built from Com and Lie via a filtered distributive law. Thus we obtain a yet another proof of the Koszulness of the associative operad, and also recover that, as an $\mathbb{S}$-module, it is isomorphic to Com $\circ$ Lie.

\subsection{Filtered distributive laws and Koszul duality}

An easy linear algebra exercise shows that if $\mathscr{E}$ is obtained from $\mathscr{A}$ and $\mathscr{B}$ via the mappings $s$ and $d$ as above, then the Koszul dual operad $\mathscr{E} !$ is similarly obtained from $\mathscr{B} !$ and $\mathscr{A} !$. The following result shows that the notion of a filtered distributive law agrees very well with the Koszul duality theory for operads (which our previous example - being Koszul self-dual - did not quite manifest).

Theorem 5.4 Assume that the operad $\mathscr{E}$ is obtained from the binary quadratic operads $\mathscr{A}$ and $\mathscr{B}$ via a filtered distributive law. Then its Koszul dual $\mathscr{E} !$ is obtained from $\mathscr{B} !$ and $\mathscr{A}^{!}$by a filtered distributive law as well whenever the projection $\mathscr{E} ! \rightarrow \mathscr{B} !$ splits.

Proof If both operads $\mathscr{A}$ and $\mathscr{B}$ are Koszul, then $\mathscr{E}$ is Koszul, and this gives us enough information to complete the proof; see [10] for details. Let us give a proof in the case of arbitrary $\mathscr{A}$ and $\mathscr{B}$ to show a yet another application of methods developed in [13].

Let us define an ordering on tree monomials in the free shuffle operad generated by $\mathscr{V}^{f} \oplus \mathscr{W}^{f}$ in the following way. For two tree monomials, we first compute the number of generators from $\mathscr{V} f$ used in each of them; if for one of them that number is greater than for the other, we say that monomial is greater than the other. Otherwise, we compare tree monomials using the lexicographic ordering on paths (Dotsenko and Khoroshkin [12], and Dotsenko and Vejdemo-Johansson [14]). This way we can be 
sure that the leading monomials of $\mathscr{R}^{f}$, tree monomials spanning $\mathscr{W}^{f} \bullet \mathscr{V}^{f}$ and the leading monomials of $\mathscr{S}^{f}$, are the leading monomials of the defining relations of $\mathscr{E}$.

Since the $\mathbb{S}$-module $\mathscr{E}$ is a quotient of $\mathscr{A} \circ \mathscr{B}$, the distributive law condition ensures that the set of weight- 3 leading monomials of the reduced Gröbner basis of $\mathscr{E} f$ is the union of the set of weight- 3 leading monomials of the reduced Gröbner basis of $\mathscr{A}^{f}$ and the set of weight- 3 leading monomials of the reduced Gröbner basis of $\mathscr{B}^{f}:$ the presence of "mixed" leading monomials would make $\mathscr{E}_{(3)}$ smaller than its natural upper bound $(\mathscr{A} \circ \mathscr{B})_{(3)}$. In other words, all S-polynomials [12] of weight 3 of $\mathscr{E} f$ are either S-polynomials of $\mathscr{A}^{f}$ or S-polynomials of $\mathscr{B}^{f}$.

The above description of leading monomials of the reduced Gröbner basis means that we have the full information on the part of the free resolution of $\mathscr{E} f$ consisting of elements of weight at most 3 , and a simple description of the homology classes of the bar complex of $\mathscr{E} f$ up to weight 3 . From [13], we know that generators of a free resolution of $\mathscr{E} f$ can be constructed in terms of "overlaps" of leading monomials of the reduced Gröbner basis of $\mathscr{E} f$. Such generators of weight 2 are precisely the leading monomials of the defining relations, whereas the generators of weight 3 are either overlaps of pairs of leading monomials of defining relations or leading monomials of weight- 3 elements of the reduced Gröbner basis. The differential induced on the space of the generators of that free resolution can be computed as follows. If an overlap of two leading monomials of defining relations produces, according to Buchberger's algorithm [12], a nontrivial $\mathrm{S}$-polynomial, the differential maps the generator corresponding to that overlap to the generator corresponding to the leading term of the respective $\mathrm{S}$-polynomial. Otherwise, the differential maps the corresponding generator to zero. Together with the information on S-polynomials of $\mathscr{E} f$ that we have, this means that up to weight 3 the homology of the bar complex of $\mathscr{E} f$ is isomorphic to the shuffle composition of the corresponding homology for $\mathscr{B}^{f}$ and $\mathscr{A}^{f}$. Since the Koszul dual operads are dual to the diagonal parts of the bar homology, our statement follows in the shuffle category. In the symmetric category, we observe that because of the splitting of $\mathscr{E} ! \rightarrow \mathscr{B} !$, there is a surjection $\mathscr{B} ! \circ \mathscr{A}^{!} \rightarrow \mathscr{E} !$, and its bijectivity in weight 3 in the shuffle category implies bijectivity in the symmetric category as well.

\subsection{Operadic Künneth formula}

We conclude this section with a general observation, which appears to be useful for transferring statements of the characteristic zero operad theory in positive characteristic. If one examines the proof of Theorem 5.2 carefully, it becomes obvious that it works because of the following statement, a particular case of the operadic Künneth formula [28], which is valid over any ground field $\mathbb{k}$. 
Theorem 5.5 Let $\mathscr{M}$ and $\mathscr{N}$ be two reduced differential graded $\mathbb{S}$-modules. Then

$$
H_{*}(\mathscr{M} \circ \mathscr{N}) \simeq H_{*}(\mathscr{M}) \circ H_{*}(\mathscr{N}) .
$$

Proof Let us note that there is a natural map

$$
\kappa: H_{*}(\mathscr{M}) \circ H_{*}(\mathscr{N}) \rightarrow H_{*}(\mathscr{M} \circ \mathscr{N}) .
$$

Our strategy is to apply the forgetful functor, and prove that

$$
\kappa^{f}:\left(H_{*}(\mathscr{M}) \circ H_{*}(\mathscr{N})\right)^{f} \rightarrow\left(H_{*}(\mathscr{M} \circ \mathscr{N})\right)^{f}
$$

is an isomorphism in the shuffle category. Since the forgetful functor is one-to-one on objects, this would mean that $\kappa$ is an isomorphism. In the shuffle category, since the forgetful functor is monoidal (that is the only part of the proof where it is crucial that our collections are reduced), we have

$$
\begin{aligned}
\left(H_{*}(\mathscr{M}) \circ H_{*}(\mathscr{N})\right)^{f} & \simeq\left(H_{*}(\mathscr{M})\right)^{f} \circ_{\mathrm{sh}}\left(H_{*}(\mathscr{N})\right)^{f} \\
& \simeq H_{*}\left(\mathscr{M}^{f}\right) \circ_{\mathrm{sh}} H_{*}\left(\mathscr{N}^{f}\right),
\end{aligned}
$$

Finally, since the shuffle composition is polynomial in the components of $\mathscr{M}^{f}$ and $\mathscr{N}^{f}$, we have

$$
H_{*}\left(\mathscr{M}^{f} \circ_{\mathrm{sh}} \mathscr{N}^{f}\right) \simeq H_{*}\left(\mathscr{M}^{f}\right) \circ_{\mathrm{sh}} H_{*}\left(\mathscr{N}^{f}\right),
$$

and the theorem follows.

\section{Koszulness of cacti and other operads}

In this section, we prove that the operads $\mathrm{NAP}_{D}$ and $\mathrm{BCACT}_{C}$ are Koszul, and also show how one can use filtered distributive laws to recover known results, and obtain new results on the structure of various known operads.

\subsection{The operad PostLie}

The operad PostLie was defined and studied in Chapoton and Vallette [8; 34], and recently appeared in various contexts; see Bai, Bellier, Guo and Ni [4; 3], and Burde, Dekimpe and Vercammen [6;5]. It is generated by a skew-symmetric operation $[\cdot, \cdot]$ and an operation $\cdot \circ \cdot$ without any symmetries that satisfy the relations

$$
\begin{gathered}
{[a,[b, c]]+[b,[c, a]]+[c,[a, b]]=0,} \\
(a \circ b) \circ c-a \circ(b \circ c)-(a \circ c) \circ b+a \circ(c \circ b)=a \circ[b, c], \\
{[a, b] \circ c=[a \circ c, b]+[a, b \circ c] .}
\end{gathered}
$$


The Koszul dual operad PostLie ${ }^{!}=$ComTrias of commutative trialgebras is generated by a symmetric operation $\cdot \bullet \cdot$ and an operation $\cdot \star \cdot$ without any symmetries that satisfy the relations

$$
\begin{aligned}
(a \star b) \star c & =a \star(b \star c)=a \star(c \star b), \\
(a \bullet b) \bullet c & =a \bullet(b \bullet c), \\
a \star(b \star c) & =a \star(b \bullet c), \\
a \bullet(b \star c) & =(a \bullet b) \star c .
\end{aligned}
$$

The magmatic operad Mag is freely generated by one binary operation without any symmetries.

Theorem 6.1 The operad PostLie is Koszul, and as an $\mathbb{S}$-module is isomorphic to Lie $\circ$ Mag.

Proof By an immediate computation, we see that the operad PostLie is built from the operads $\mathscr{A}=$ Lie and $\mathscr{B}=$ Mag via a filtered distributive law. Indeed, we may put $\mathscr{V}=\operatorname{span}([\cdot, \cdot]), \mathscr{W}=\operatorname{span}(\cdot \circ \cdot)$, and

$$
\begin{gathered}
s([a,[b, c]]+[b,[c, a]]+[c,[a, b]])=0, \\
d([a, b] \circ c)=[a \circ c, b]+[a, b \circ c], \\
d(a \circ[b, c])=(a \circ b) \circ c-a \circ(b \circ c)-(a \circ c) \circ b+a \circ(c \circ b)
\end{gathered}
$$

(the weight- 3 condition can be easily checked by hand, and since $s=0$, the projection is split automatically). This proves both statements of our theorem.

The Koszulness of PostLie and PostLie! = ComTrias was established in [8] using partition posets. Note that our approach applies to ComTrias as well, since the splitting of the projection ComTrias $\rightarrow \mathrm{Mag}^{!}=$Nil only requires the splitting on the level of generators, which we already have. The $\mathbb{S}$-module isomorphism PostLie $\simeq$ Lie $\circ$ Mag was first observed in [34]. ${ }^{1}$ This isomorphism, together with the following corollary, allows to complete the PostLie algebras description in Zinbiel [36].

Corollary 6.2 The suboperad of PostLie generated by $\cdot \circ \cdot$ is isomorphic to Mag.

Note that the dual version of this corollary is not true: even though on the level of $\mathbb{S}$-modules we have ComTrias $\simeq$ Nil $\circ \mathrm{Com}$, it is easy to check the suboperad of the operad ComTrias generated by the operation $\cdot \star \cdot$ is isomorphic to Perm.

\footnotetext{
${ }^{1}$ The proof in the published version of that paper is incomplete (one has to check that the extension of .$\circ \cdot$ to the free algebra $\operatorname{Lie}(\operatorname{Mag}(V))$ is consistent with the Jacobi identity).
} 


\subsection{The operad of commutative tridendriform algebras}

The operad of commutative tridendriform algebras was studied by Loday [27]. Let us write down the relations of this operad, and of its Koszul dual. The operad CTD is generated by a symmetric operation $\cdot \star \cdot$ and an operation $\cdot \prec \cdot$ without any symmetries that satisfy the relations

$$
\begin{gathered}
(a \prec b) \prec c=a \prec(b \prec c+c \prec b+b \star c), \\
(a \star b) \prec c=a \star(b \prec c), \\
(a \star b) \star c=a \star(b \star c) .
\end{gathered}
$$

The operad CTD! is generated by a skew-symmetric operation $[\cdot, \cdot]$ and an operation •• without any symmetries that satisfy the relations

$$
\begin{gathered}
{[a,[b, c]]+[b,[c, a]]+[c,[a, b]]=0,} \\
a \bullet[b, c]=a \bullet(b \bullet c), \\
{[a, b] \bullet c=[a \bullet c, b]+[a, b \bullet c],} \\
(a \bullet b) \bullet c=a \bullet(b \bullet c)+(a \bullet c) \bullet b .
\end{gathered}
$$

Theorem 6.3 - The operad CTD is Koszul, and as an $\mathbb{S}$-module is isomorphic to Zinb $\circ$ Com .

- The operad CTD! is Koszul, and as an $\mathbb{S}-$ module is isomorphic to Lie o Leib.

Proof By an immediate computation, we notice that the operad CTD is built from the operad Zinb and Com via a filtered distributive law. Indeed, we may put $\mathscr{V}=$ $\operatorname{span}(\cdot \prec \cdot), \mathscr{W}=\operatorname{span}(\cdot \star \cdot)$, and

$$
\begin{gathered}
s((a \prec b) \prec c-a \prec(b \prec c+c \prec b))=a \prec(b \star c), \\
d(a \star(b \prec c))=(a \star b) \prec c .
\end{gathered}
$$

(the weight- 3 condition can be easily checked by hand; the projection CTD $\rightarrow$ Zinb splits because $\operatorname{Zinb}(n)$ is a free $\mathbb{S}_{n}$-module). Therefore Theorems 5.2 and 5.4 prove all the statements of our theorem (for the latter, we observe that the projection $C T D^{!} \rightarrow$ Lie splits because for CTD! we have $s=0$ ).

The $\mathbb{S}$-module isomorphism in the first part was proved in [27] as a consequence of the existence of a good triple of operads (As, CTD, Com) and the isomorphism of $\mathbb{S}$ modules As $\simeq$ Zinb. Our results recover that isomorphism, prove a similar isomorphism for CTD!, and also describe the sub-operads of CTD and CTD! generated by either one of the operations. This provides the following bits of information that have been missing in [36]. 
Proposition 6.4 - The generating series of the operad of dual commutative tridendriform algebras is equal to

$$
f^{\mathrm{CTD}^{!}}(t)=-\log \left(\frac{1-2 t}{1-t}\right)
$$

- The suboperad of CTD! generated by the operation - • • is isomorphic to Leib.

Note that though the underlying $\mathbb{S}$-module of the operad Zinb $=$ Leib! is used in the definition of the operad CTD, the dual statement to the second part of this proposition is not true: in the operad CTD, the suboperad generated by $\cdot \prec \cdot$ is not isomorphic to Zinb because of the "lower term" $a \prec(b \star c)$ added to the Zinbiel relation.

\subsection{The linear NAP ${ }_{D}$ operad}

Proposition 6.5 Let $D$ be a graded vector space. The operad NAP $\mathrm{N}_{D}$ is generated by binary operations $D \otimes \mathbb{K S}_{2}$; these operations satisfy the relations

$$
d^{\prime} \circ_{1} d^{\prime \prime} .(23)=(-1)^{\left|d^{\prime}\right|\left|d^{\prime \prime}\right|} d^{\prime \prime} \circ_{1} d^{\prime} \quad\left(\text { for homogeneous } d^{\prime}, d^{\prime \prime} \in D\right) \text {. }
$$

Proof The "geometric" version of this proposition is proved as part of Proposition 3.1. That the linear version is generated by binary operations may be proved by precisely the same method. As before, the relations just express the symmetric group action on trees:

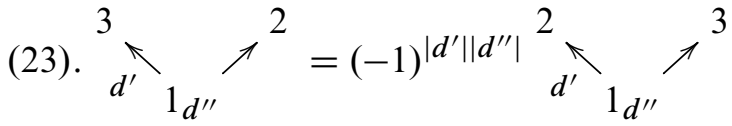

Theorem 6.6 The operad $\mathrm{NAP}_{D}$ is Koszul.

Proof Note that according to Proposition 6.5, the operad NAP $_{D}$ is a quotient of the operad $\mathscr{N}_{D}$ generated by binary operations $D \otimes \mathbb{k} \mathbb{S}_{2}$ subject only to relations (6.3.1). Let us show that the operad $\mathscr{N}_{D}$ is Koszul, and is isomorphic to $\mathrm{NAP}_{D}$.

First of all, one can easily check that the Koszul dual $\mathscr{N}_{D}^{!}$of the operad $\mathscr{N}_{D}$ has generators $D^{*} \otimes \mathbb{k S}_{2}$ subject to relations

$$
\begin{gathered}
e^{\prime} \circ_{1} e^{\prime \prime}=(-1)^{\left|e^{\prime}\right|\left|e^{\prime \prime}\right|} e^{\prime \prime} \circ_{1} e^{\prime} .(23) \quad\left(\text { for homogeneous } e^{\prime}, e^{\prime \prime} \in D^{*}\right), \\
e^{\prime} \circ_{2} e^{\prime \prime}=0 .
\end{gathered}
$$

This immediately implies that if we choose a basis $e_{1}, \ldots, e_{n}$ of $D^{*}$, then for a basis of NAP! ${ }_{D}(1)$ we can take the set of all "left combs"

$$
\left(e_{i_{1}} \circ_{1} e_{i_{2}} \circ_{1} \cdots \circ_{1} e_{i_{n-1}}\right) .(1, k, k-1, \ldots, 2),
$$


because our relations mean that the tree monomials can only "grow" to the left, and that we can reorder all elements except for the leftmost one arbitrarily. There are $(\operatorname{dim} D)^{n-1} \cdot n$ such monomials. At the same time, if we explicitly write the relations of $\mathscr{N}_{D}$ as a shuffle operad, we see that its relations are

$$
\begin{aligned}
d^{\prime} \circ_{1} d^{\prime \prime} .(23) & =(-1)^{\left|d^{\prime}\right|\left|d^{\prime \prime}\right|} d^{\prime \prime} \circ_{1} d^{\prime}, \\
d^{\prime} \circ_{1} \tilde{d}^{\prime \prime} . & (23)=(-1)^{\left|d^{\prime}\right|\left|d^{\prime \prime}\right|}{\tilde{d^{\prime \prime}}}_{\circ_{2}} \tilde{d}^{\prime}, \\
d^{\prime} \circ_{1} \tilde{d}^{\prime \prime} & =(-1)^{\left|d^{\prime}\right|\left|d^{\prime \prime}\right|}{\tilde{d^{\prime \prime}}}_{j} \circ_{2} d^{\prime} .
\end{aligned}
$$

Here we use the notation $\tilde{d}$ to abbreviate the "opposite operation" $d \otimes \sigma \in D \otimes \mathbb{k} \mathbb{S}_{2}$.

Let us pick a basis $d_{1}, \ldots, d_{n}$ of $D$, and define an ordering of tree monomials in the free shuffle operad with binary generators $D \otimes \mathbb{k S}_{2}$ that is very similar to the pathlexicographic ordering [12]. For two tree monomials, we first compare lexicographically their sequences of leaves, read left-to-right, and then compare the path sequences of those monomials, assuming

$$
d_{1}<\cdots<d_{n}<d_{1} .(12)<\cdots<d_{n} .(12) .
$$

The leading monomials of the relations of $\mathscr{N}_{D}$ are, respectively, $d_{i} \circ_{1} d_{j}$.(23), $d_{i} \circ_{1}$ $\tilde{d}_{j}$.(23) and $d_{i} \circ_{1} \tilde{d}_{j}$. The trees built from these monomials as building blocks give an upper bound on the dimensions of components of the Koszul dual operad, which is sharp precisely when our operads have quadratic Gröbner bases [11]. It is easy to see that there are exactly $(\operatorname{dim} D)^{n-1} \cdot n$ tree monomials built from these, so both the operads $\mathscr{N}_{D}$ and $\mathscr{N}_{D}^{!}$are Koszul. The power series inversion equation for Koszul operads [17] implies that

$$
f_{\mathscr{N}_{D} !}\left(-f_{\mathscr{N}_{D}}(-t)\right)=t
$$

Since it is clear that

$$
f_{\mathscr{N}_{D}^{!}}(t)=\sum_{n \geq 1} \frac{(\operatorname{dim} D)^{n-1}}{n !} t^{n}
$$

after denoting

$$
g(s):=\frac{f_{\mathscr{N}_{D}}(\operatorname{dim} D \cdot s)}{\operatorname{dim} D},
$$

we see that $g(-s)$ is the inverse of $-s \exp (-s)$ under composition, and hence $g(s)$ is the generating function enumerating rooted trees. Recalling that $\operatorname{NAP}_{D}$ as an $\mathbb{S}$ module is described as $D$-decorated rooted trees, we conclude that components of $\mathscr{N}_{D}$ and $\mathrm{NAP}_{D}$ have same dimensions, and therefore these operads are isomorphic, the former being a quotient of the latter. 
This proof concluded by showing that $\mathrm{NAP}_{D}$ is presented by quadratic relations. By considering the linearisation of the operad $\mathrm{NAP}_{F}$ when $F$ is a finite set, we see that $\mathrm{NAP}_{F}$ is also presented by quadratic relations. Now suppose that $Y$ is infinite. Any finite set of $Y$-trees involves a finite number of labels $F$ and hence any relation in $\mathrm{NAP}_{Y}$ is contained within $\mathrm{NAP}_{F}$, which is in turn presented by quadratic relations. Therefore we have the following.

Corollary 6.7 Let $Y$ be a set. Then the operad NAP $_{Y}$ is generated by binary operations and is presented by its quadratic relations.

Remark 6.8 The proof of Theorem 6.6 used arguments involving the Koszul dual and its Hilbert series to show that the quadratic relations suffices to present $\mathrm{NAP}_{Y}$. A more direct proof is possible using a certain "geometric" map from $\mathscr{F}\left(\mathrm{NAP}_{Y}(2)\right)$ to $\mathrm{NAP}_{Y}$. We will denote elements of $\operatorname{NAP}_{Y}(2)$ by

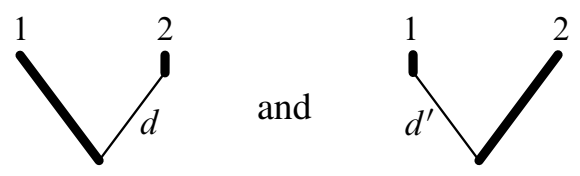

for $d, d^{\prime} \in Y$. In each generator there is a thin line labelled with an element of $Y$, a thick line running from root to the end of a leaf and a small portion of thick line at the end of the other leaf. Then the $\mathrm{NAP}_{Y}$-relation (3.1.5) states that

(6.3.13) $d^{\prime} \circ_{1} d=$
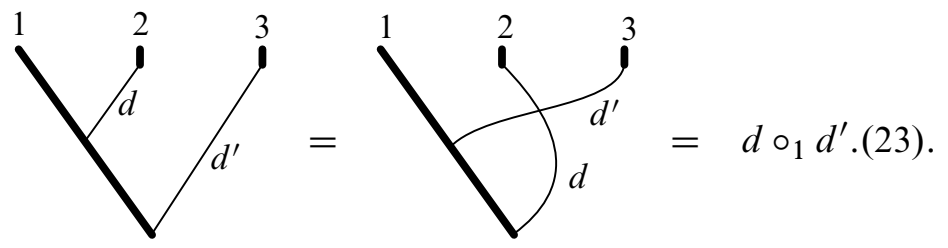

The thin lines may be seen to "move freely" along the thick lines. A couple of facts are apparent about any arity $n$ tree monomial in these generators:

(1) The thick lines never branch and each thick line can be followed up the tree to a unique leaf, in this way the thick lines are in bijection with the leaves.

(2) Every thin line joins two thick lines and is labelled by an element of $Y$.

So by contracting each thick line to a point and using these as vertices, we are left with a tree with vertex set $[n]$. The thin lines become the edges and are already labelled by elements of $Y$. This tree is rooted by following the thick line starting at the bottom of the tree monomial to its leaf. Hence we have an explicit map from $\mathscr{F}\left(\operatorname{NAP}_{Y}(2)\right)$ to 
$\mathrm{NAP}_{Y}$. Below is an example.
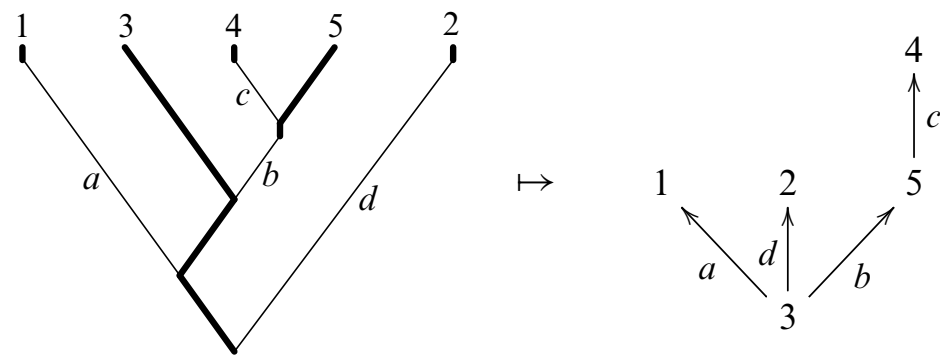

The fact that the quadratic presentation forms a Gröbner basis means that the operad it presents may be described by certain admissible tree monomials. By comparing this basis with the $Y$-trees via the map just described, we may see that $\mathrm{NAP}_{Y}$ is presented by the quadratic basis. A reader interested in combinatorics should compare our construction with one of the well known "Catalan bijections", which takes a planar rooted binary tree with $n$ leaves and contracts all left-going edges, thus obtaining a planar rooted tree with $n$ vertices.

Remark 6.9 An alternative proof of Theorem 6.6 follows from one of the results of [31]. Namely, it turns out that the operad $\mathrm{NAP}_{D}$ is isomorphic to the operad of rooted trees enriched in the monoid $S^{\bullet}(\widehat{D})$, where $\widehat{D}$ is the symmetric collection whose only non-zero component is in arity 1 and is equal to $D$, and $S^{\bullet}$ denotes the free commutative monoid in symmetric collections with respect to the tensor product (we want to emphasise that we are using the tensor product, not the composition product!). One of the main results of [31] states that the operad of rooted trees enriched in a monoid $M$ is Koszul if and only if $M$ is Koszul. The free commutative monoid is manifestly Koszul, and the theorem follows.

\subsection{The linear operads of based cacti}

Proposition 6.10 Let $(C, \Delta, \epsilon, \gamma)$ be a graded augmented cocommutative coalgebra. The operad $\mathrm{BCACT}_{C}$ is generated by binary operations $C \otimes \mathbb{k}_{2}$; these operations satisfy the relations

$$
\begin{aligned}
c^{\prime} \circ_{1} c^{\prime \prime} .(23) & =(-1)^{\left|c^{\prime}\right|\left|c^{\prime \prime}\right|} c^{\prime \prime} \circ_{1} c^{\prime} \quad\left(\text { for homogeneous } c^{\prime}, c^{\prime \prime} \in C\right), \\
c \circ_{2} \mathbb{1} & =\sum c_{(1)} \circ_{1} c_{(2)} \quad(\text { for } c \in C),
\end{aligned}
$$

which suffice to present the operad.

Proof According to Proposition 4.4, the operad $\mathrm{BCACT}_{C}$ is isomorphic to the quotient of $\mathrm{NAP}_{C}$ by the operadic ideal generated by relations (6.4.2). Also, from the proof of 
Theorem 6.6, we know that the relations (6.4.1) are the defining relations of $\mathrm{NAP}_{C}$, which completes the proof.

Remark 6.11 For the sake of completeness, let us describe the relations of the Koszul dual operad $\mathrm{BCACT}_{C}^{!}$. Its space of generators is $C^{*} \otimes \mathrm{kS}_{2}$; note that $C^{*}$ is a graded commutative algebra that splits as $\mathbb{k} \mathbb{1} \oplus \overline{C^{*}}$. The relations are

$$
c \circ_{2} \bar{c}=0 \text { for homogeneous } c \in C^{*}, \bar{c} \in \overline{C^{*}},
$$

(6.4.4) $\quad c^{\prime} \circ_{1} c^{\prime \prime}-\left(c^{\prime} c^{\prime \prime}\right) \circ_{2} \mathbb{1}$

$$
=(-1)^{\left|c^{\prime}\right|\left|c^{\prime \prime}\right|}\left(c^{\prime \prime} \circ_{1} c^{\prime}-\left(c^{\prime \prime} c^{\prime}\right) \circ_{2} \mathbb{1}\right) .(23) \text { for homogeneous } c^{\prime}, c^{\prime \prime} \in C^{*} \text {. }
$$

Note that for $c=c^{\prime}=\mathbb{1}$ the relation (6.4.4) is precisely the pre-Lie relation. This is not at all surprising, since by combining Theorem 5.4 with Theorem 6.12 below we expect that the $\mathbb{S}$-modules

$$
\mathrm{BCACT}_{C}^{!} \text {and } \mathrm{NAP}_{\bar{C}}^{!} \circ(\text { Perm })^{!} \simeq \mathrm{NAP}_{\bar{C}}^{!} \circ \text { PreLie }
$$

are isomorphic, and that PreLie is a suboperad of $\mathrm{BCACT}_{C}^{!}$.

Theorem 6.12 For a graded augmented cocommutative coalgebra $C$, the operad BCACT $_{C}$ is Koszul, and as $\mathbb{S}$-modules,

$$
\mathrm{BCACT}_{C} \simeq \operatorname{Perm}_{\mathrm{NAP}}{ }_{\bar{C}}
$$

Proof Let us show that $\mathrm{BCACT}_{C}$ is obtained from Perm and $\mathrm{NAP}_{\bar{C}}$ via a filtered distributive law.

Using the splitting of $C$ along the augmentation, we can refine the formulae (6.4.1) and (6.4.2) as

$$
\begin{aligned}
\mathbb{1} \circ_{1} \mathbb{1} .(23) & =\mathbb{1} \circ_{1} \mathbb{1}, & & \\
c \circ_{1} \mathbb{1} & =\mathbb{1} \circ_{1} c .(23) & & (\text { for } c \in \bar{C}), \\
c^{\prime} \circ_{1} c^{\prime \prime} .(23) & =(-1)^{\left|c^{\prime}\right|\left|c^{\prime \prime}\right|} c^{\prime \prime} \circ_{1} c^{\prime} & & \left(\text { for homogeneous } c^{\prime}, c^{\prime \prime} \in \bar{C}\right), \\
\mathbb{1} \circ_{2} \mathbb{1} & =\mathbb{1} \circ_{1} \mathbb{1}, & & \\
c \circ_{2} \mathbb{1} & =\sum c_{(1)} \circ_{1} c_{(2)} & & (\text { for } c \in \bar{C}) .
\end{aligned}
$$

The formulae (6.4.7), (6.4.8) and (6.4.9) represent the formula (6.4.1) after splitting, and the formulae (6.4.10) and (6.4.11) represent the formula (6.4.2) after splitting. It is clear that the formulae (6.4.7) and (6.4.10) describe the operad Perm, while the formula (6.4.9) describes precisely the operad $\operatorname{NAP}_{\bar{C}}$. It remains to show that the formulae (6.4.8) and (6.4.11) define a filtered distributive law between these two 
operads. To be precise, we first need to check that the formula (6.4.11) stands a chance of defining a distributive law, since a priori its right-hand side is a mixture of all possible tree monomials. However, we first note that the compatibility of the counit with the coproduct ensures that if $c \in \bar{C}$ then

$$
\Delta(c) \in \bar{C} \otimes \mathbb{k} \mathbb{1}+\mathbb{k} \mathbb{1} \otimes \bar{C}+\bar{C} \otimes \bar{C},
$$

so the tree monomial $\mathbb{1} \circ_{1} \mathbb{1}$ is missing on the right-hand side of (6.4.11). Also, the tree monomials of the form $c^{\prime} \circ_{1} \mathbb{1}$ (with $c^{\prime} \in \bar{C}$ ) appearing on the right-hand side should be rewritten using the formula (6.4.8), but this minor detail will not affect any of our computations.

To check that the formulae (6.4.8) and (6.4.11) define a filtered distributive law between Perm and $\operatorname{NAP}_{\bar{C}}$, one needs to perform carefully all ambiguous rewritings bringing the generator $\mathbb{1}$ towards the root of a tree monomial, checking that they do not give additional new relations. We shall omit the details, indicating briefly that the rewriting of

$$
c \circ_{2}\left(\mathbb{1} \circ_{1} \mathbb{1} .(23)\right)=c \circ_{2}\left(\mathbb{1} \circ_{1} \mathbb{1}\right)
$$

does not result in a new relation because the coproduct of $C$ is cocommutative, while the rewriting of

$$
c \circ_{2}\left(\mathbb{1} \circ_{1} \mathbb{1}\right)=c \circ_{2}\left(\mathbb{1} \circ_{2} \mathbb{1}\right)
$$

does not result in a new relation because the coproduct of $C$ is coassociative, and finally the rewritings of

$$
\begin{gathered}
c^{\prime} \circ_{1}\left(c^{\prime \prime} \circ_{2} \mathbb{1}\right)=(-1)^{\left|c^{\prime}\right|\left|c^{\prime \prime}\right|}\left(c^{\prime \prime} \circ_{2} \mathbb{1}\right) \circ_{1} c^{\prime}, \\
\left(c \circ_{1} \mathbb{1}\right) \circ_{3} \mathbb{1}=\left(c \circ_{2} \mathbb{1}\right) \circ_{1} \mathbb{1}
\end{gathered}
$$

do not result in new relations because of the NAP-type relations (6.4.1). This, together with the observation that the projection $\mathrm{BCACT}_{C} \rightarrow$ Perm always splits because the relations of Perm remain undeformed $(s=0)$, completes the proof of our theorem.

Remark 6.13 Let $Y$ be the (pointed) two-element set $\{\mathbf{0}, 1\}$, so that $C=H_{*}(Y)$ is the split two-dimensional coalgebra $\mathbb{k} \oplus \mathbb{k}$, as in the Example 4.8 below. Theorem 6.12 shows that we have an $\mathbb{S}$-module isomorphism

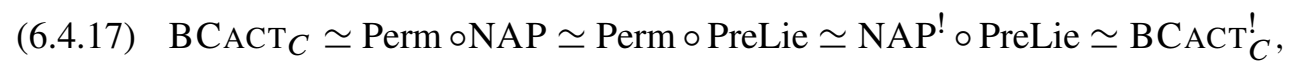

but the operads $\mathrm{BCACT}_{C}$ and $\mathrm{BCACT}_{C}^{!}$are substantially different. Of course, there is also a trivial operad structure on the $\mathbb{S}$-module Perm $\circ$ PreLie for which the insertion of any Perm-operation into any PreLie-operation is equal to zero; this operad is Koszul and self-dual. It is an open question whether there exist nontrivial self-dual Koszul 
operad structures on Perm $\circ$ PreLie via a distributive law or a filtered distributive law between Perm and PreLie; such operads would be natural candidates to encode "prePoisson algebras" (much different from the ones in Aguiar [1]) and "pre-associative algebras".

\section{References}

[1] M Aguiar, Pre-Poisson algebras, Lett. Math. Phys. 54 (2000) 263-277 MR1846958

[2] V I Arnol' d, The cohomology ring of the group of dyed braids, Mat. Zametki 5 (1969) 227-231 MR0242196

[3] C Bai, O Bellier, L Guo, X Ni, Splitting of operations, Manin products, and RotaBaxter operators, Int. Math. Res. Not. 2013 (2013) 485-524 MR3021790

[4] C Bai, L Guo, $\mathbf{X} \mathbf{N i}$, Nonabelian generalized Lax pairs, the classical Yang-Baxter equation and postLie algebras, Comm. Math. Phys. 297 (2010) 553-596 MR2651910

[5] D Burde, K Dekimpe, Post-Lie algebra structures and generalized derivations of semisimple Lie algebras, Mosc. Math. J. 13 (2013) 1-18, 189 MR3112213

[6] D Burde, K Dekimpe, K Vercammen, Affine actions on Lie groups and post-Lie algebra structures, Linear Algebra Appl. 437 (2012) 1250-1263 MR2942346

[7] F Chapoton, Un endofoncteur de la catégorie des opérades, from: "Dialgebras and related operads", (J-L Loday, F A, F Chapoton, F Goichot, editors), Lecture Notes in Math. 1763, Springer, Berlin (2001) 105-110 MR1860996

[8] F Chapoton, B Vallette, Pointed and multi-pointed partitions of type $A$ and $B, \mathrm{~J}$. Algebraic Combin. 23 (2006) 295-316 MR2236610

[9] F R Cohen, T J Lada, J P May, The homology of iterated loop spaces, Lecture Notes in Mathematics 533, Springer, New York (1976) MR0436146

[10] V Dotsenko, An operadic approach to deformation quantization of compatible Poisson brackets, I, J. Gen. Lie Theory Appl. 1 (2007) 107-115 MR2320771

[11] V Dotsenko, Freeness theorems for operads via Gröbner bases, from: "OPERADS 2009”, (J-L Loday, B Vallette, editors), Sémin. Congr. 26, Soc. Math. France, Paris (2011) 61-76

[12] V Dotsenko, A Khoroshkin, Gröbner bases for operads, Duke Math. J. 153 (2010) 363-396 MR2667136

[13] V Dotsenko, A Khoroshkin, Quillen homology for operads via Gröbner bases, Doc. Math. 18 (2013) 707-747 MR3084563

[14] V Dotsenko, M Vejdemo-Johansson, Implementing Gröbner bases for operads, from: “OPERADS 2009”, (J-L Loday, B Vallette, editors), Sémin. Congr. 26, Soc. Math. France, Paris (2013) 77-98 MR3203368 
[15] A Dzhumadil' daev, C Löfwall, Trees, free right-symmetric algebras, free Novikov algebras and identities, Homology Homotopy Appl. 4 (2002) 165-190 MR1918188

[16] E Getzler, J D S Jones, Operads, homotopy algebra, and iterated integrals for double loop spaces arXiv:hep-th/9403055

[17] V Ginzburg, M Kapranov, Koszul duality for operads, Duke Math. J. 76 (1994) 203-272 MR1301191

[18] J T Griffin, Diagonal complexes and the integral homology of the automorphism group of a free product, Proc. Lond. Math. Soc. 106 (2013) 1087-1120 MR3066749

[19] C Jensen, J McCammond, J Meier, The integral cohomology of the group of loops, Geom. Topol. 10 (2006) 759-784 MR2240905

[20] R M Kaufmann, On several varieties of cacti and their relations, Algebr. Geom. Topol. 5 (2005) 237-300 MR2135554

[21] A Khoroshkin, Koszul operads and distributive lattices, preprint ITEP-TH-24/06 (2006)

[22] J Kock, Notes on polynomial functors (2009) Available at http://mat.uab.es/ kock/cat/notes-on-polynomial-functors.html

[23] J Kock, A Joyal, M Batanin, J-F Mascari, Polynomial functors and opetopes, Adv. Math. 224 (2010) 2690-2737 MR2652220

[24] M Livernet, A rigidity theorem for pre-Lie algebras, J. Pure Appl. Algebra 207 (2006) 1-18 MR2244257

[25] J-L Loday, Une version non commutative des algèbres de Lie: Les algèbres de Leibniz, Enseign. Math. 39 (1993) 269-293 MR1252069

[26] J-L Loday, Cup-product for Leibniz cohomology and dual Leibniz algebras, Math. Scand. 77 (1995) 189-196 MR1379265

[27] J-L Loday, On the algebra of quasi-shuffles, Manuscripta Math. 123 (2007) 79-93 MR2300061

[28] J-L Loday, B Vallette, Algebraic operads, Grundl. Math. Wissen. 346, Springer, Heidelberg (2012) MR2954392

[29] M Markl, Distributive laws and Koszulness, Ann. Inst. Fourier (Grenoble) 46 (1996) 307-323 MR1393517

[30] J P May, Simplicial objects in algebraic topology, 2nd edition, University of Chicago Press (1992) MR1206474

[31] M A Méndez, Koszul duality for monoids and the operad of enriched rooted trees, Adv. in Appl. Math. 44 (2010) 261-297 MR2593312

[32] I Moerdijk, On the Connes-Kreimer construction of Hopf algebras, from: "Homotopy methods in algebraic topology”, (J P C Greenlees, R Bruner, N Kuhn, editors), Contemp. Math. 271, Amer. Math. Soc. (2001) 311-321 MR1831357 
[33] I Moerdijk, E Palmgren, Wellfounded trees in categories, Ann. Pure Appl. Logic 104 (2000) 189-218 MR1778939 From "Proceedings of the Workshop on Proof Theory and Complexity, PTAC'98” (C Butz, U Kohlenbach, S Riis, G Winskel, editors), Aarhus (1998)

[34] B Vallette, Homology of generalized partition posets, J. Pure Appl. Algebra 208 (2007) 699-725 MR2277706

[35] B Vallette, A Koszul duality for PROPs, Trans. Amer. Math. Soc. 359 (2007) 4865-4943 MR2320654

[36] G W Zinbiel, Encyclopedia of types of algebras 2010, from: "Operads and universal algebra”, (C Bai, L Guo, J-L Loday, editors), Nankai Ser. Pure Appl. Math. Theoret. Phys. 9, World Sci. Publ., Hackensack, NJ (2012) 217-297 MR3013090

School of Mathematics, Trinity College Dublin

College Green, Dublin 2, Ireland

School of Mathematics and Statistics, University of Glasgow

University Gardens, Glasgow G12 8QW, UK

vdots@maths.tcd.ie, James.Griffin@glasgow.ac.uk

Received: 10 November $2011 \quad$ Revised: 5 March 2014 
